\title{
Visible-light-mediated oxidative coupling of vinylarenes with bromocarboxylates leading to Y-ketoesters
}

Xing-Xiao Fang, ${ }^{\dagger}$ Peng-Fei Wang, ${ }^{\dagger}$ Wei $\mathrm{Yi}^{\dagger}{ }^{\dagger}$ Wei Chen, Sheng-Chun Lou and Gong-Qing Liu*

School of Pharmacy, Nantong University, 19 Qixiu Road, Nantong 226001, People's Republic of China

${ }^{\dagger}$ These authors are contributed equally.

E-mail: gqliu@ntu.edu.cn

\section{Content}

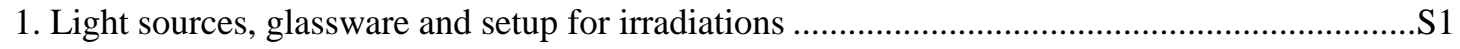

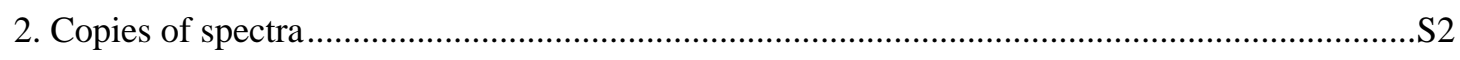




\section{Light sources, glassware and setup for irradiations}

\section{$\underline{\text { Light sources }}$}

All photoreactions were performed using a $24 \mathrm{~W}$ energy-saving household CFL bulb (cool daylight, $6500 \mathrm{~K}$ ) by Opple. Please refer to the website (https://detail.tmall.com/item.htm?id=36296589905\&spm=a1z09.2.0.0.289f2e8dLvL PIS\&_u=nkj7u3r52c7) for more detail.

\section{Glassware}

All reactions were performed in $25 \mathrm{~mL}$ vials made of Synthware. For detailed technical information, the reader is directed to the homepage of Synthware: http://www.xinweier.com/.

\section{Setup for irradiations}

All photoreactions with iridium-based catalysts were performed with the $24 \mathrm{~W}$ energy-saving CFL-bulb introduced above. The reaction vessel (a $25 \mathrm{~mL}$ Schlenk tube sealed with a rubber septum) was placed approximately $2 \mathrm{~cm}$ from the light source. A typical reaction setup is shown in Figure S1.
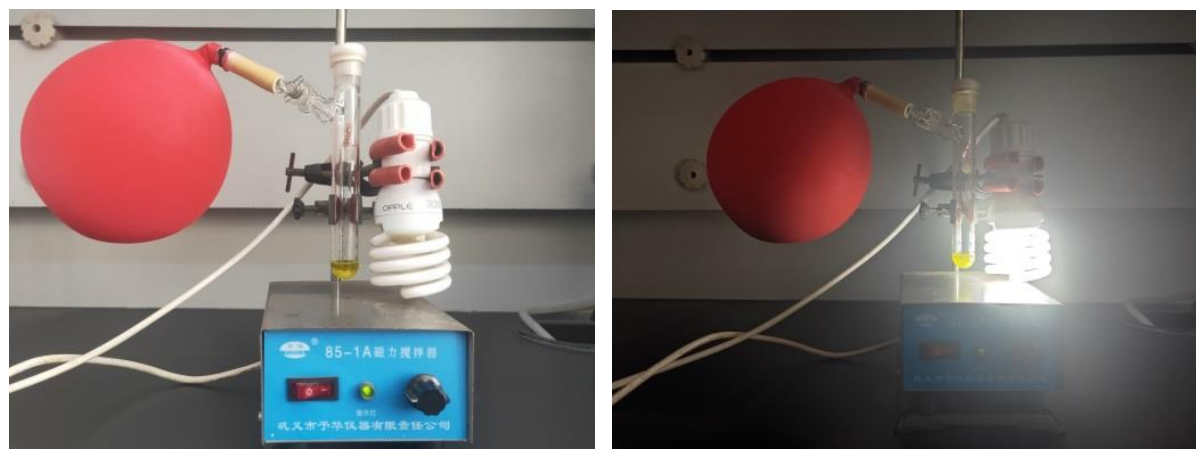

Figure S1. Photograph of the photocatalytic reactor used for reactions 


\section{Copies of spectra}

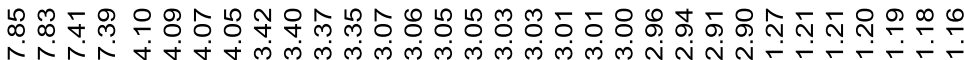

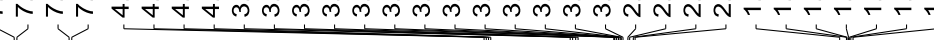

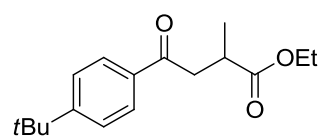

${ }^{1} \mathrm{H}$ NMR $\left(400 \mathrm{MHz}, \mathrm{CDCl}_{3}\right)$ of $\mathbf{3 a}$

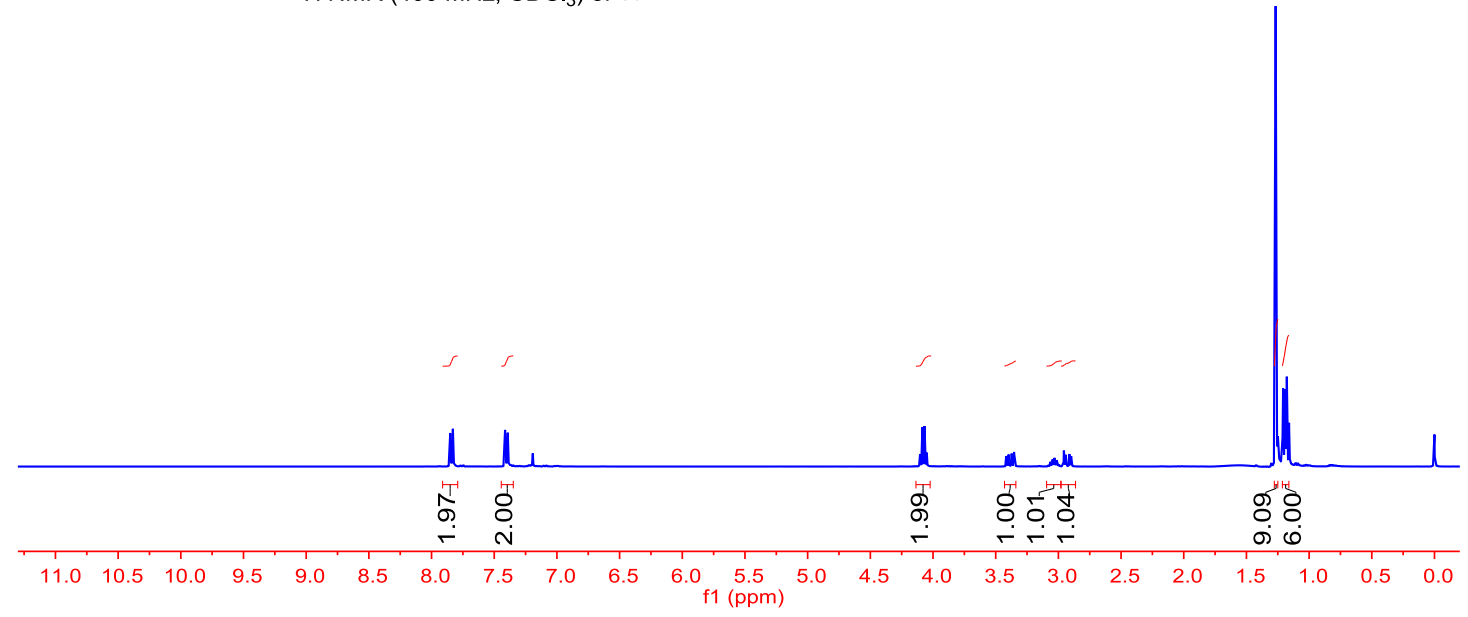

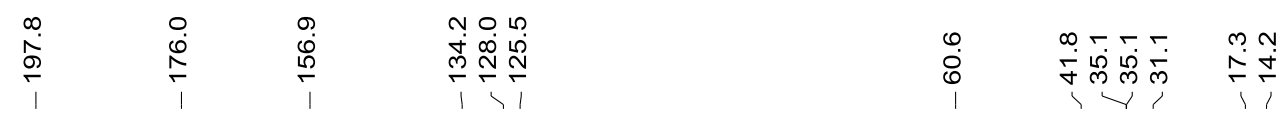

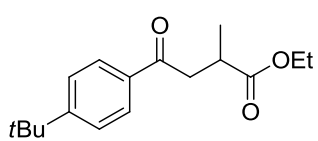

${ }^{13} \mathrm{C} \mathrm{NMR}\left(100 \mathrm{MHz}, \mathrm{CDCl}_{3}\right)$ of $\mathbf{3 a}$

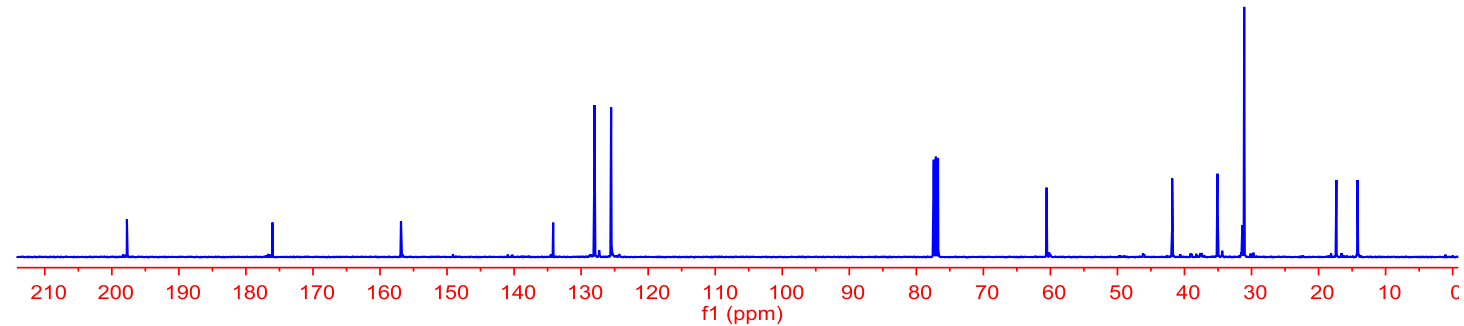




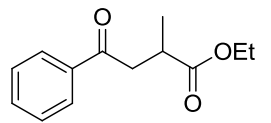

${ }^{1} \mathrm{H} \mathrm{NMR}\left(400 \mathrm{MHz}, \mathrm{CDCl}_{3}\right)$ of $\mathbf{3 b}$

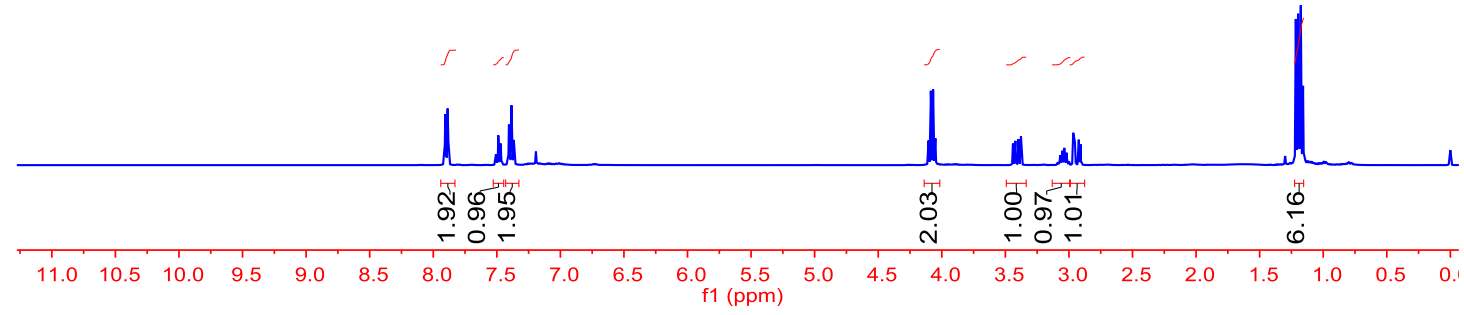

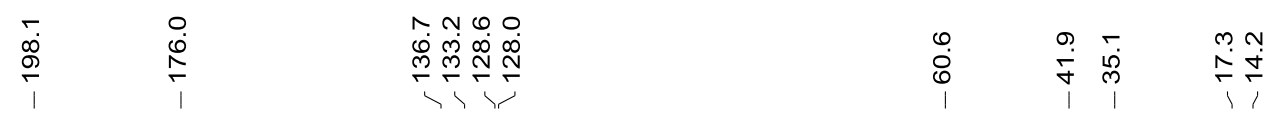

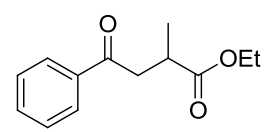

${ }^{13} \mathrm{C} \mathrm{NMR}\left(100 \mathrm{MHz}, \mathrm{CDCl}_{3}\right)$ of $\mathbf{3 b}$

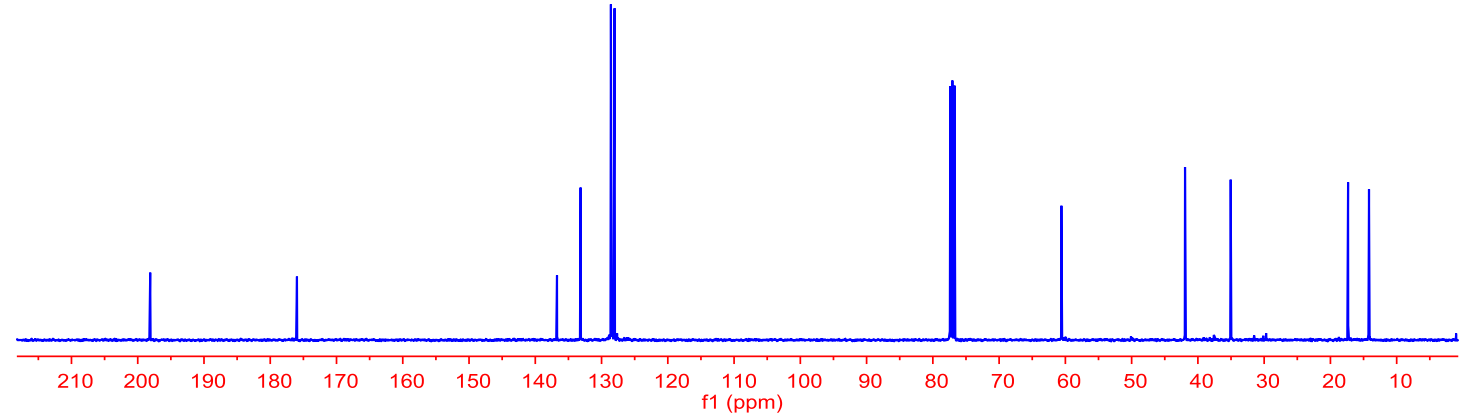




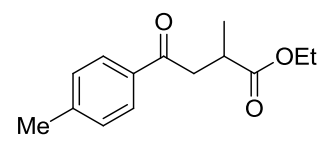

${ }^{1} \mathrm{H}$ NMR $\left(400 \mathrm{MHz}, \mathrm{CDCl}_{3}\right)$ of $\mathbf{3 c}$
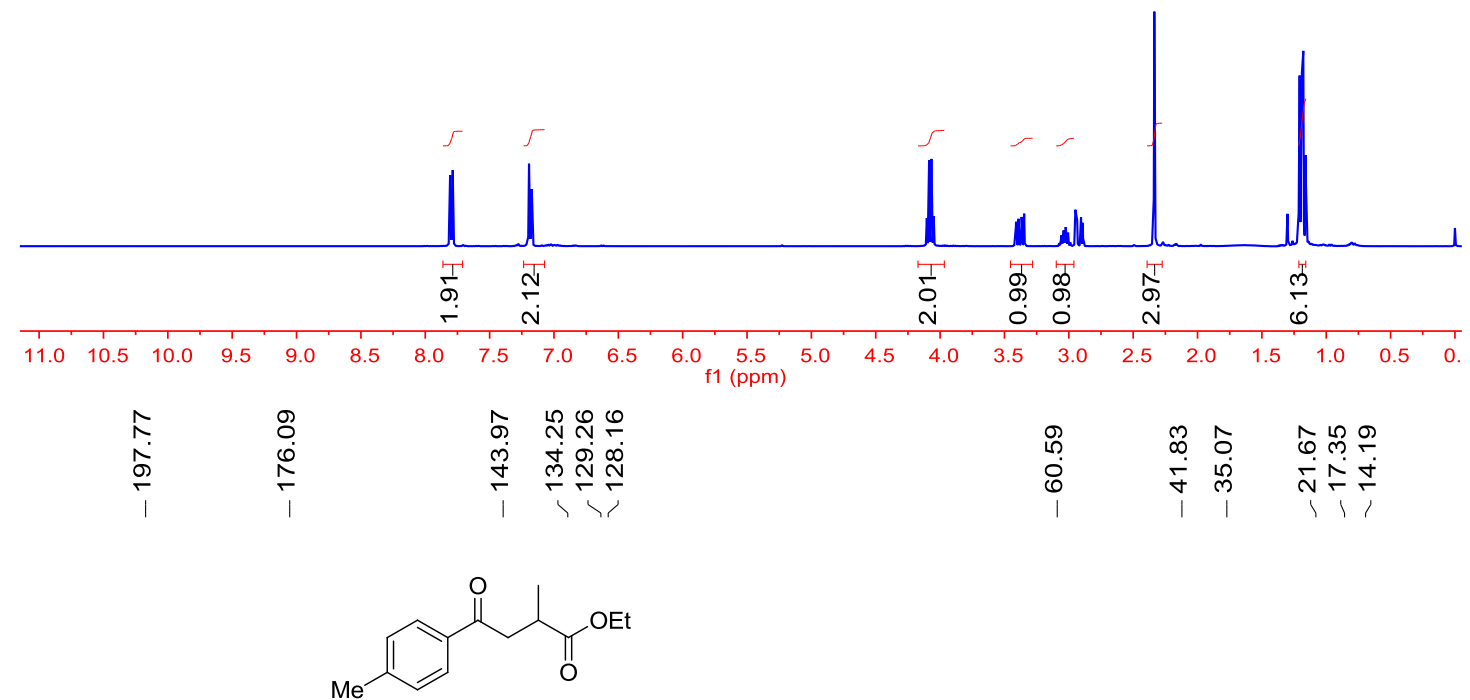

${ }^{13} \mathrm{C} \mathrm{NMR}\left(100 \mathrm{MHz}, \mathrm{CDCl}_{3}\right)$ of $3 \mathrm{c}$

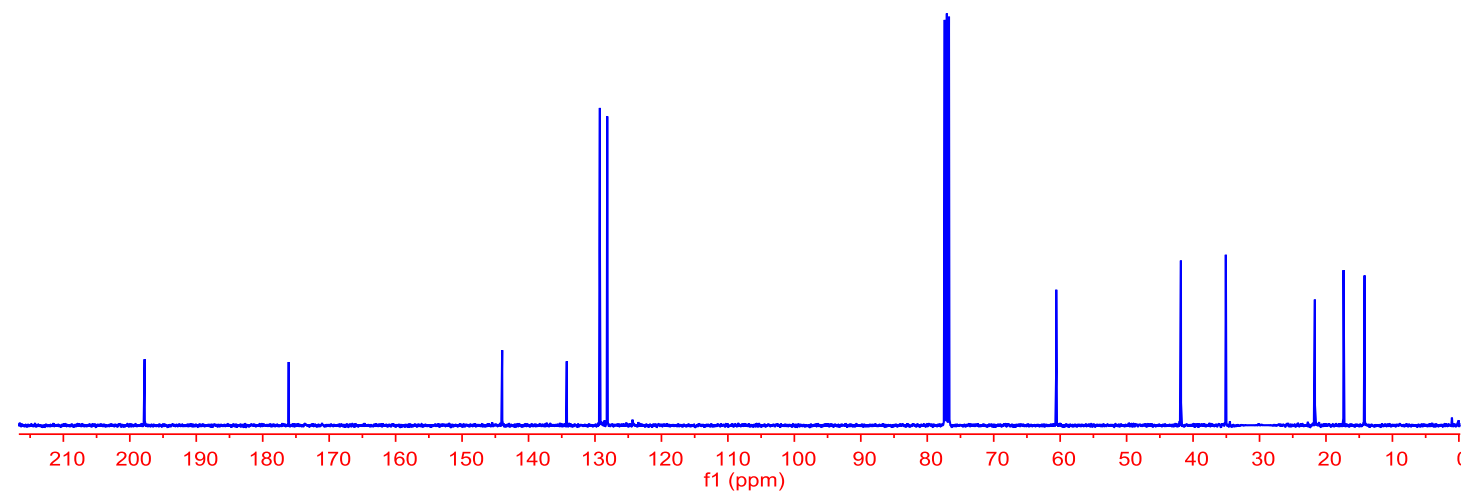




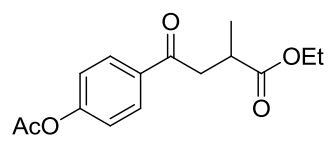

${ }^{1} \mathrm{H} \mathrm{NMR}\left(400 \mathrm{MHz}, \mathrm{CDCl}_{3}\right)$ of $\mathbf{3 d}$
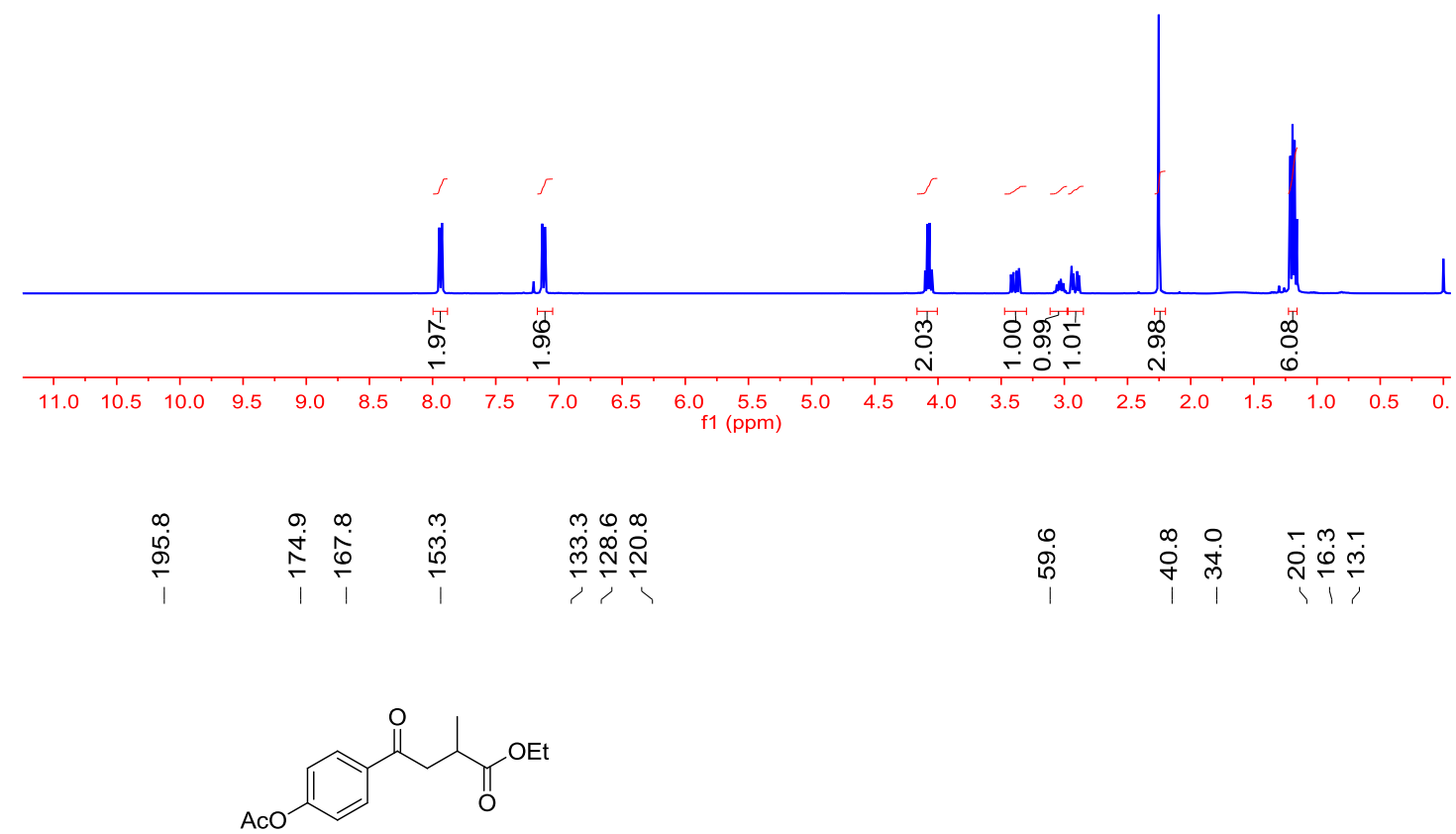

${ }^{13} \mathrm{C} \mathrm{NMR}\left(100 \mathrm{MHz}, \mathrm{CDCl}_{3}\right)$ of $\mathbf{3 d}$

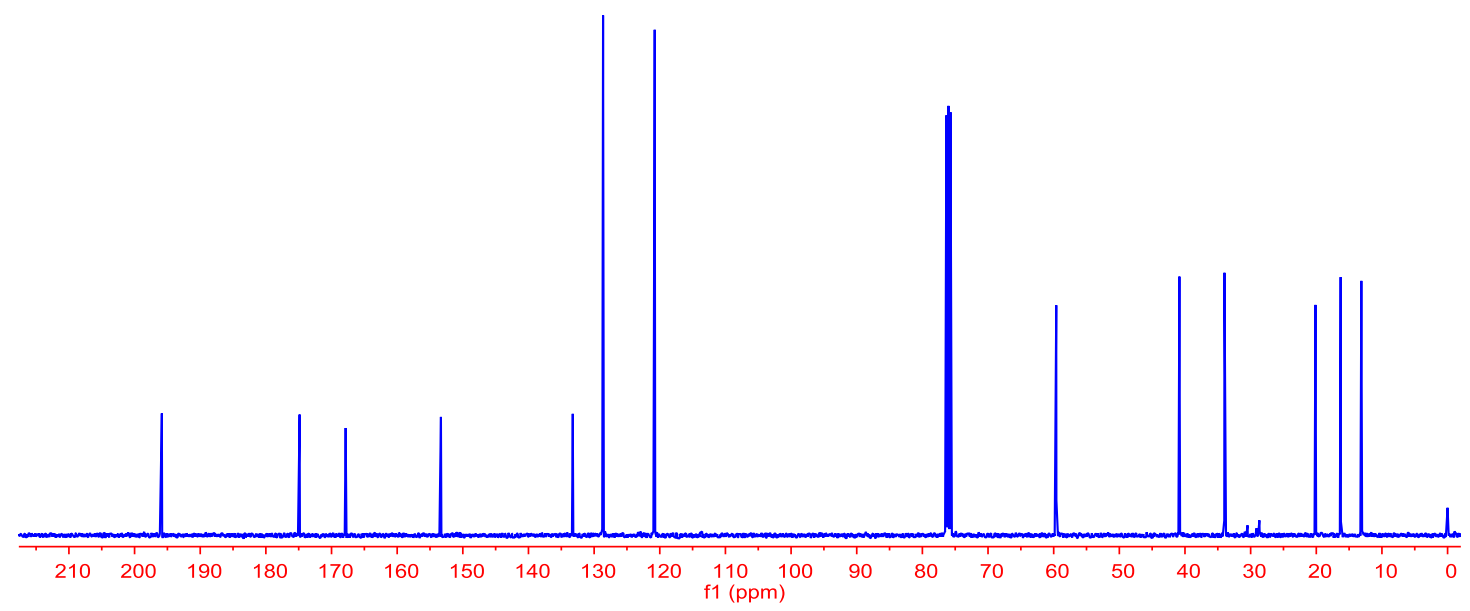




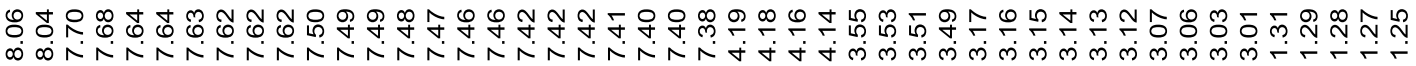<smiles>CCOC(=O)C(C)CC(=O)c1ccc(-c2ccccc2)cc1</smiles>

${ }^{1} \mathrm{H} \mathrm{NMR}\left(400 \mathrm{MHz}, \mathrm{CDCl}_{3}\right)$ of $3 e$

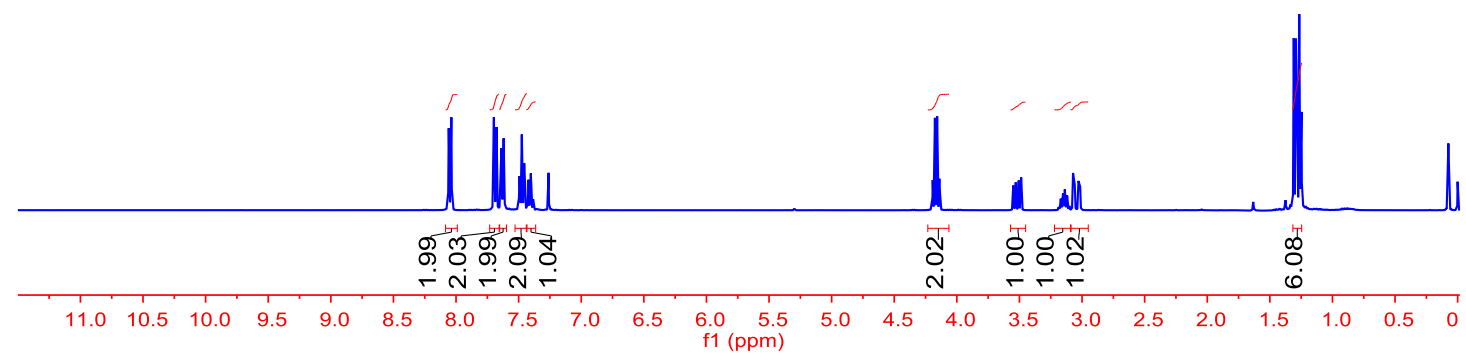

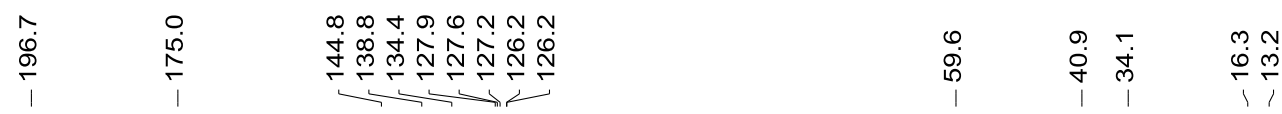<smiles>CCOC(=O)C(C)CC(=O)c1ccc(-c2ccccc2)cc1</smiles>

${ }^{13} \mathrm{C} \mathrm{NMR}\left(100 \mathrm{MHz}, \mathrm{CDCl}_{3}\right)$ of $3 \mathbf{e}$

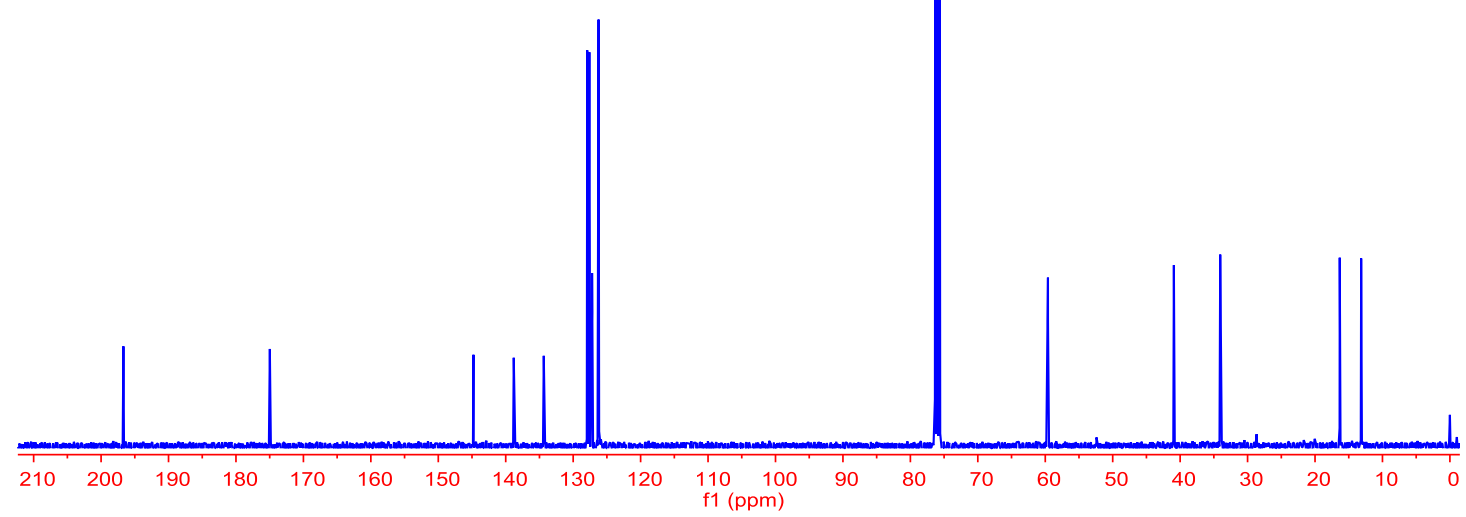




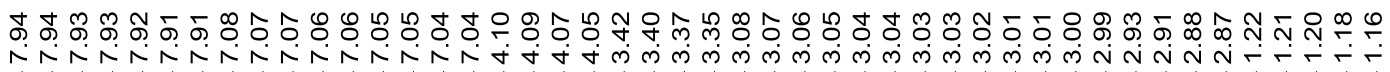<smiles>CCOC(=O)C(C)CC(=O)c1ccc(F)cc1</smiles>

${ }^{1} \mathrm{H}$ NMR $\left(400 \mathrm{MHz}, \mathrm{CDCl}_{3}\right)$ of $3 \mathrm{f}$
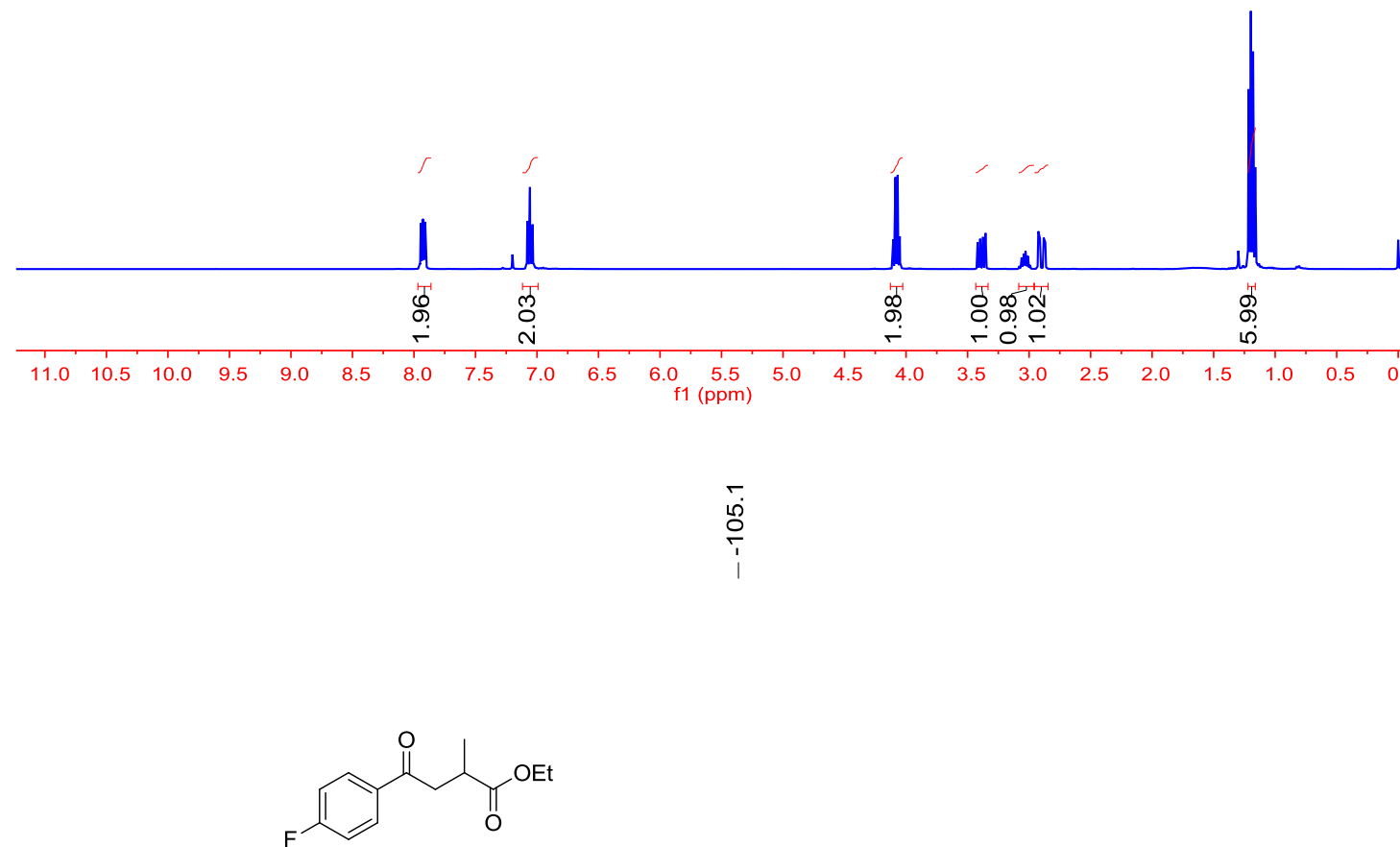

${ }^{19} \mathrm{~F} \mathrm{NMR}\left(376 \mathrm{MHz}, \mathrm{CDCl}_{3}\right)$ of $\mathbf{3 f}$

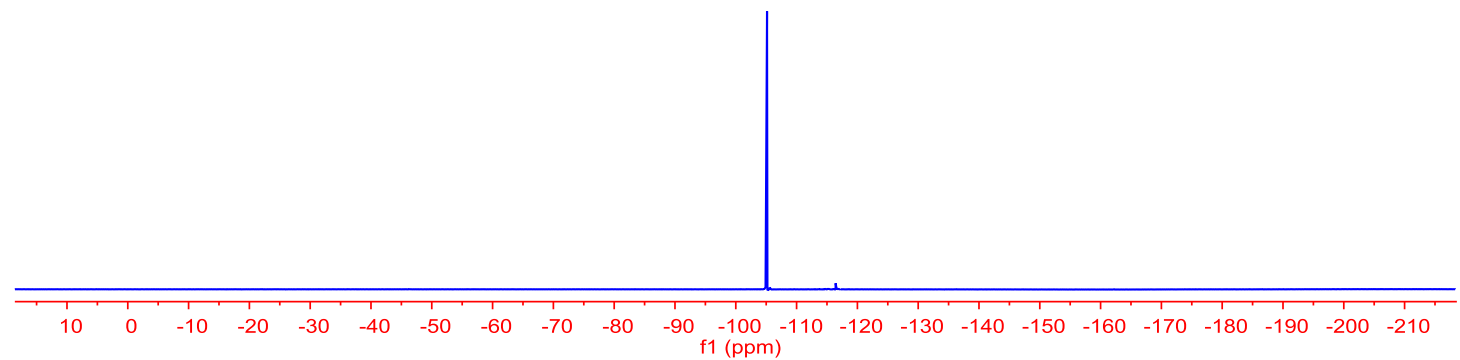




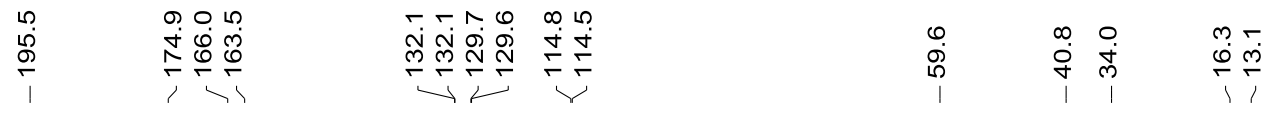

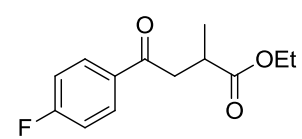

${ }^{13} \mathrm{C} \mathrm{NMR}\left(100 \mathrm{MHz}, \mathrm{CDCl}_{3}\right)$ of $\mathbf{3 f}$

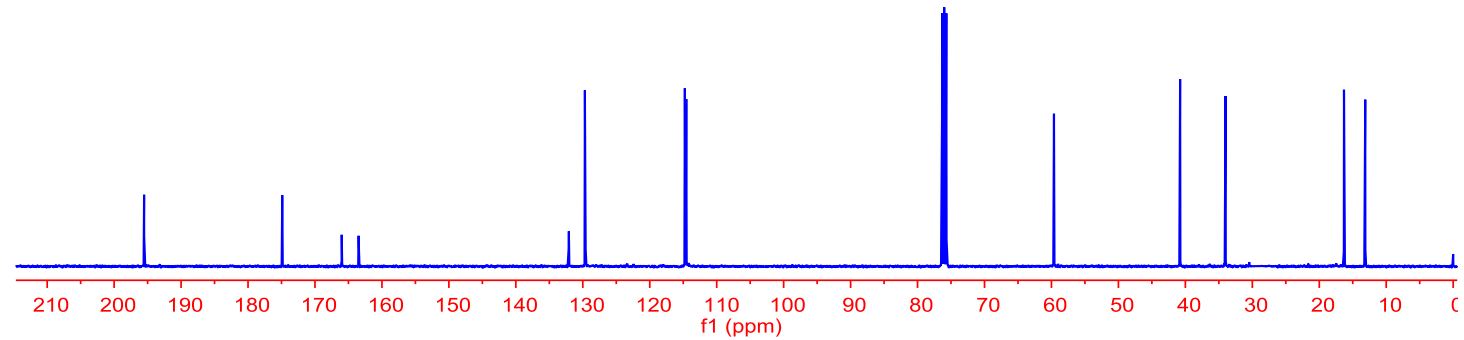

ఏ싰

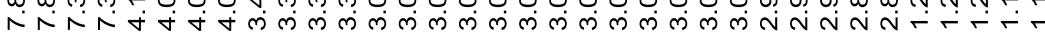<smiles>CCOC(=O)C(C)CC(=O)c1ccc(Cl)cc1</smiles>

${ }^{1} \mathrm{H} \mathrm{NMR}\left(400 \mathrm{MHz}, \mathrm{CDCl}_{3}\right)$ of $\mathbf{3 g}$

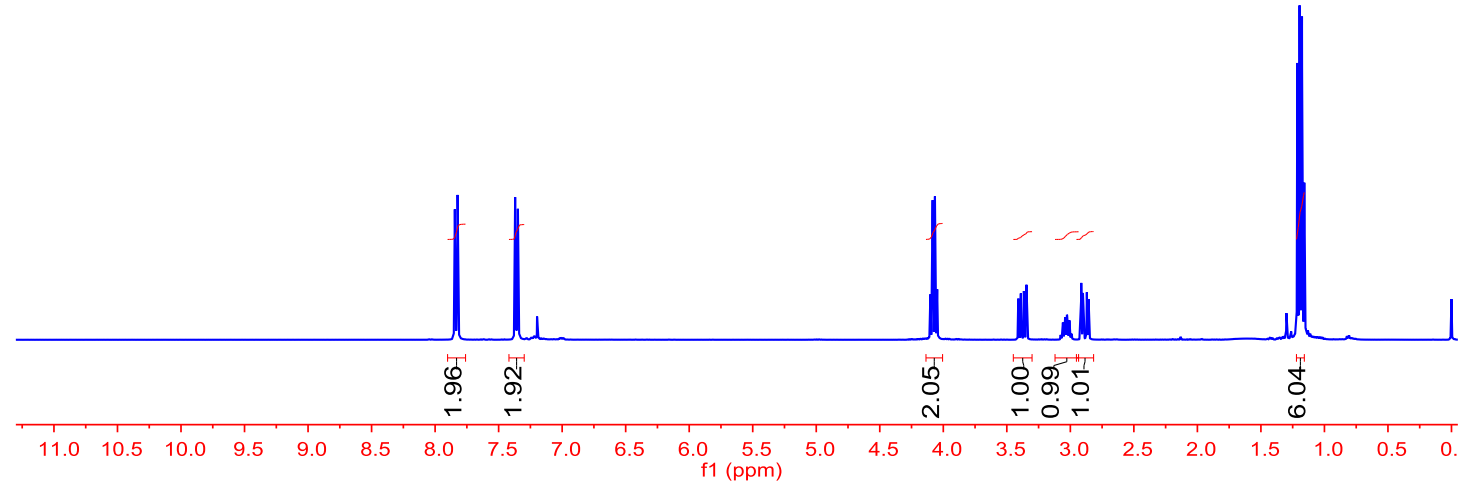




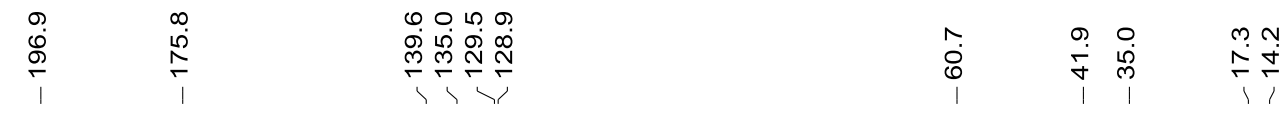<smiles>CCOC(=O)C(C)CC(=O)c1ccc(Cl)cc1</smiles>

${ }^{13} \mathrm{C} \mathrm{NMR}\left(100 \mathrm{MHz}, \mathrm{CDCl}_{3}\right)$ of $\mathbf{3 g}$

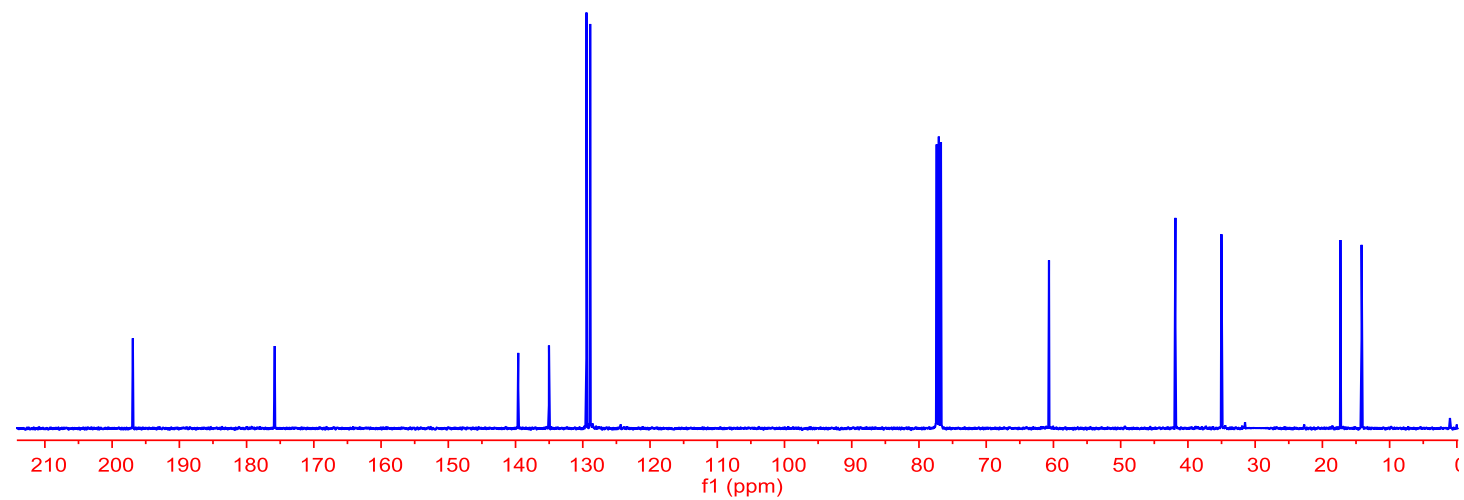

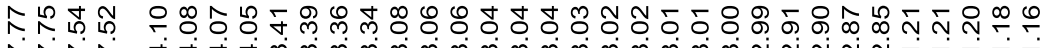

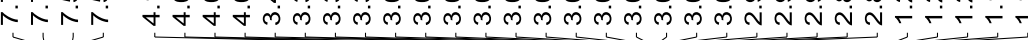<smiles>CCOC(=O)C(C)CC(=O)c1ccc(Br)cc1</smiles>

${ }^{1} \mathrm{H} \mathrm{NMR}\left(400 \mathrm{MHz}, \mathrm{CDCl}_{3}\right)$ of $\mathbf{3 h}$

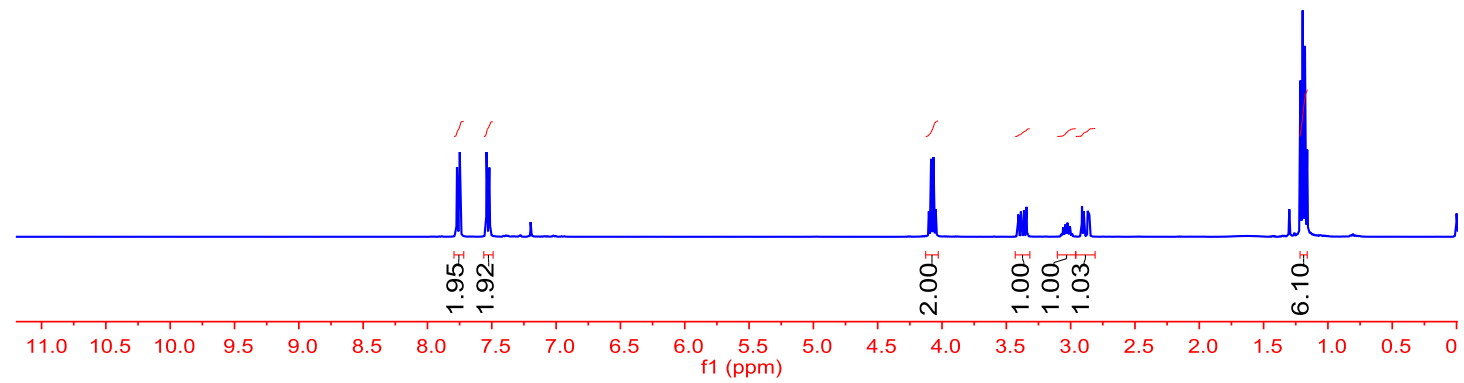




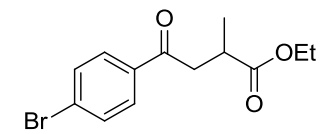

$\left.{ }^{13} \mathrm{C} \mathrm{NMR} \mathrm{(100} \mathrm{MHz,} \mathrm{CDCl}_{3}\right)$ of $\mathbf{3 h}$

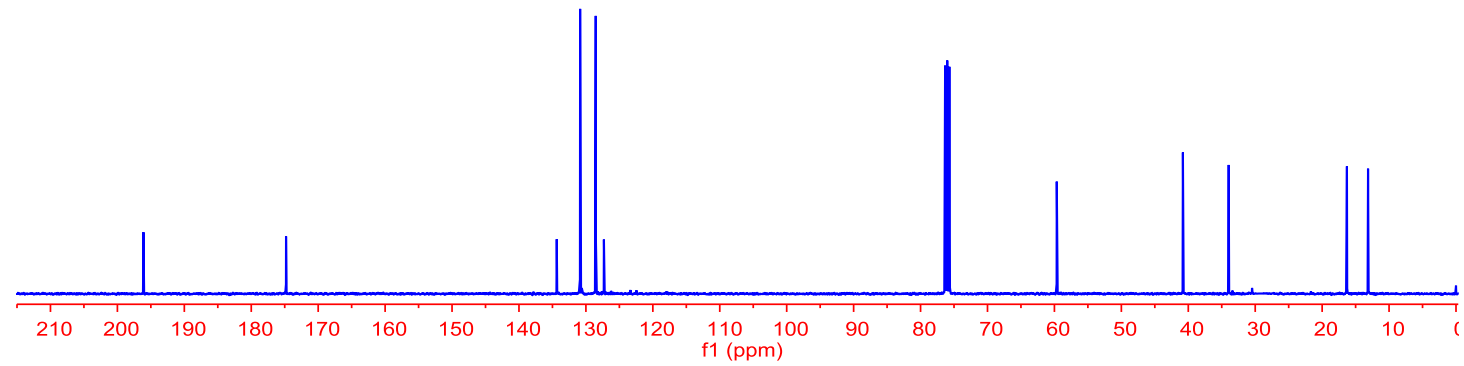

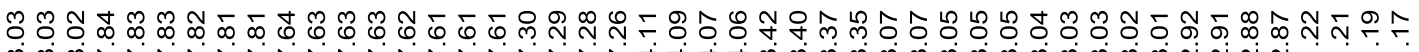

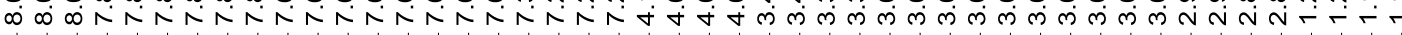<smiles>CCOC(=O)C(C)CC(=O)c1cccc(Br)c1</smiles>

${ }^{1} \mathrm{H} \mathrm{NMR}\left(400 \mathrm{MHz}, \mathrm{CDCl}_{3}\right)$ of $\mathbf{3 i}$

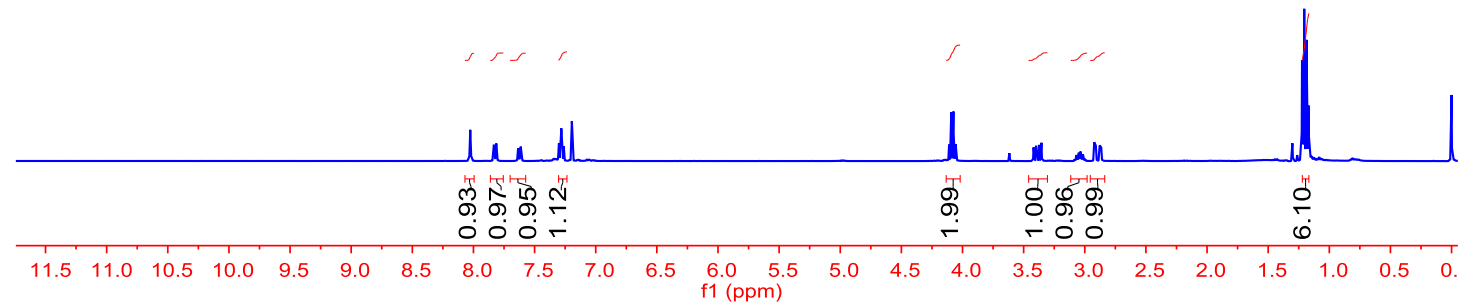




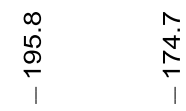
ᄀ.
ஸेm

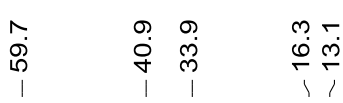

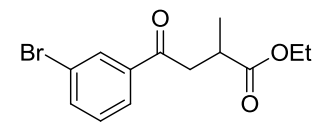

${ }^{13} \mathrm{C} \mathrm{NMR} \mathrm{(100} \mathrm{MHz,} \mathrm{CDCl}_{3}$ ) of $\mathbf{3 i}$

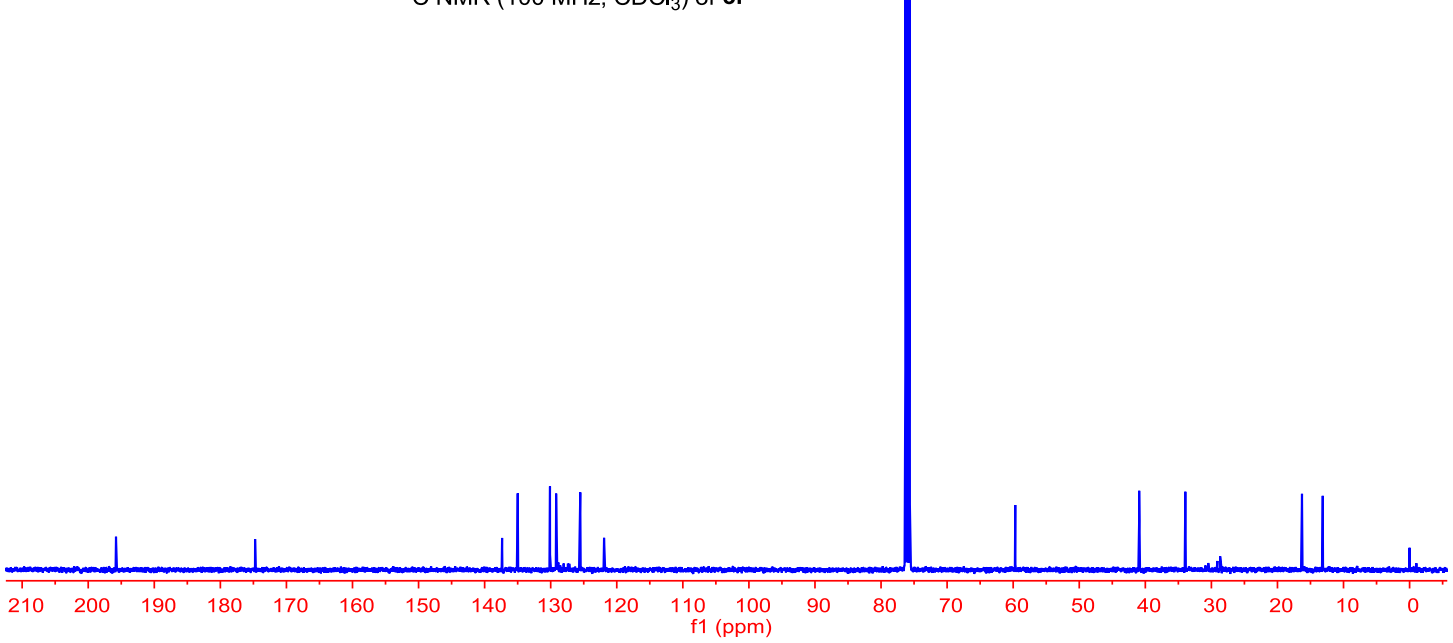

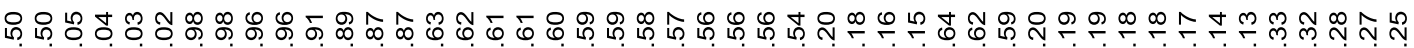

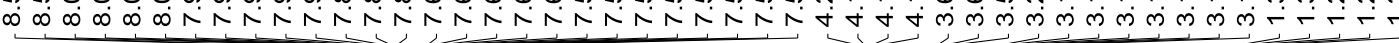

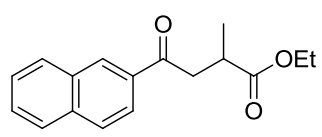

${ }^{1} \mathrm{H} \mathrm{NMR}\left(400 \mathrm{MHz}, \mathrm{CDCl}_{3}\right)$ of $\mathbf{3 j}$

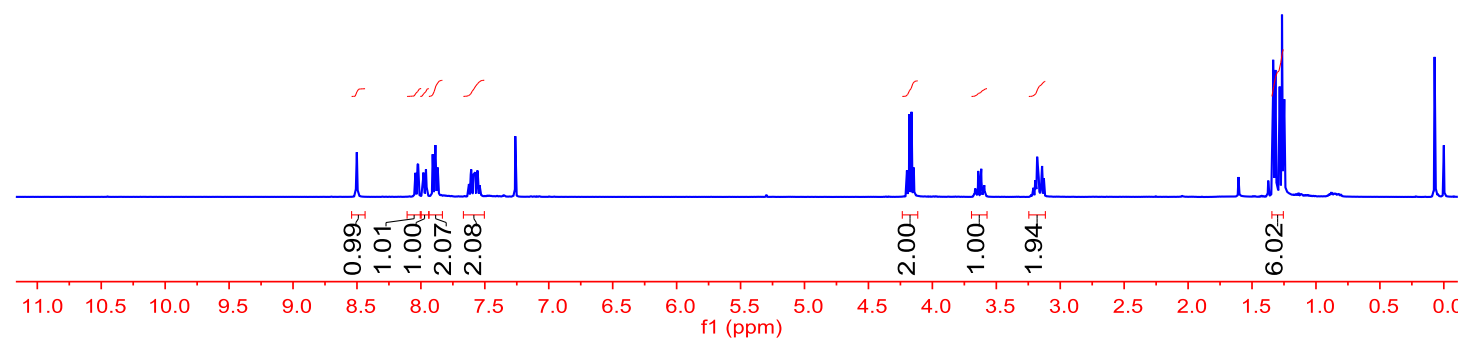




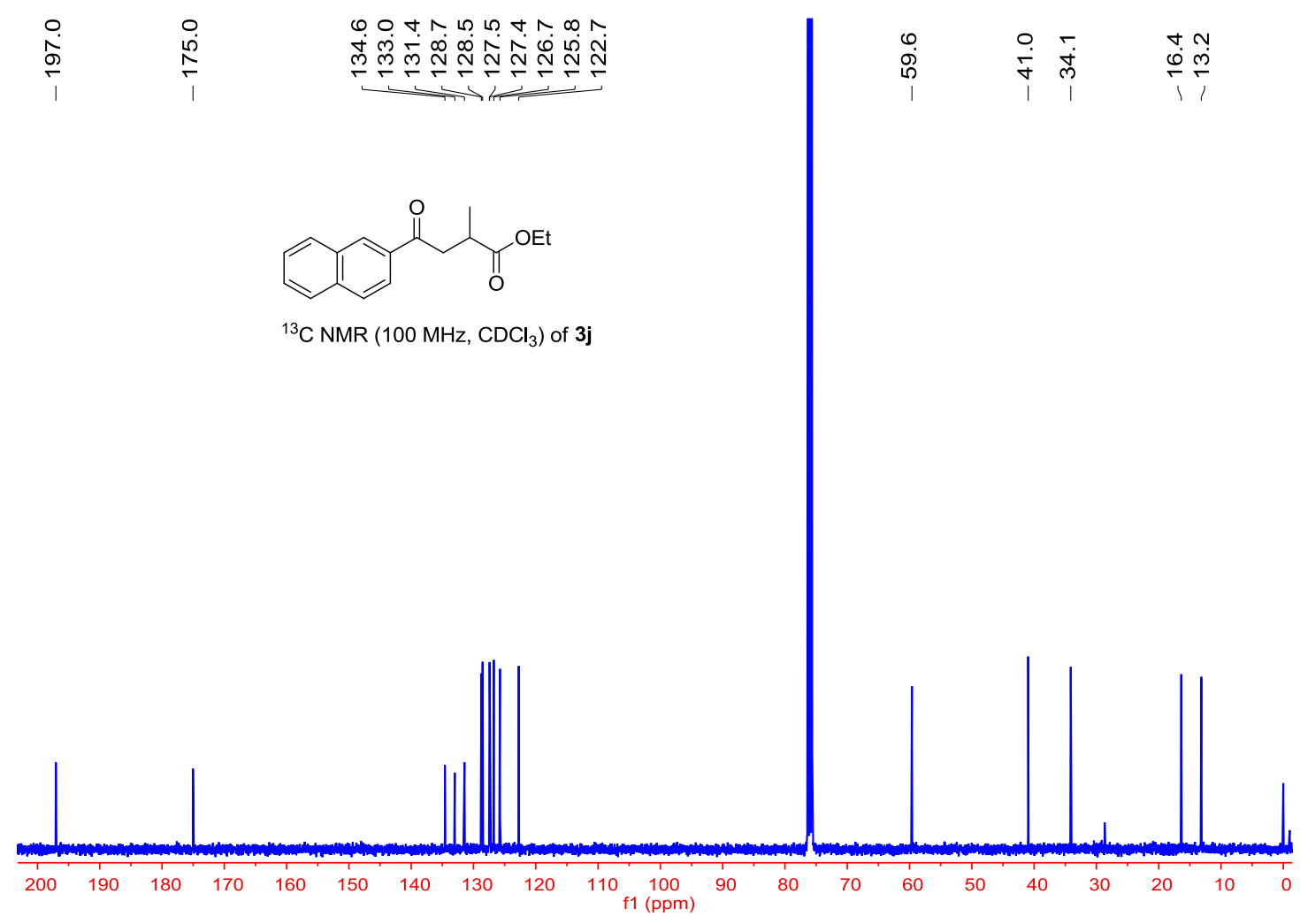

Љ

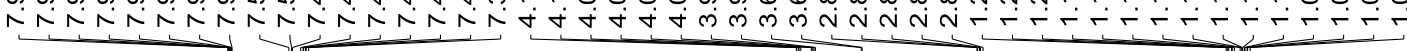<smiles>CCOC(=O)C(C)C(C)C(=O)c1ccccc1</smiles>

${ }^{1} \mathrm{H}$ NMR $\left(400 \mathrm{MHz}, \mathrm{CDCl}_{3}\right)$ of $\mathbf{3 k}$

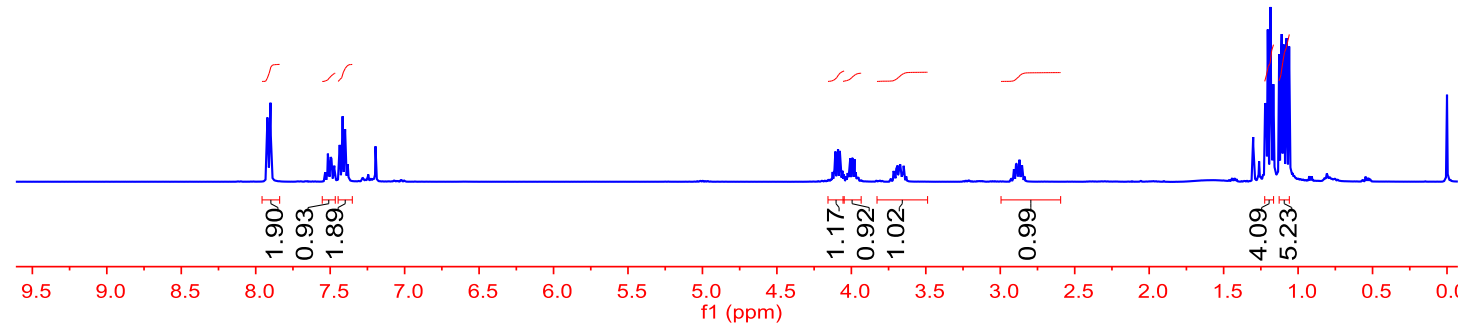




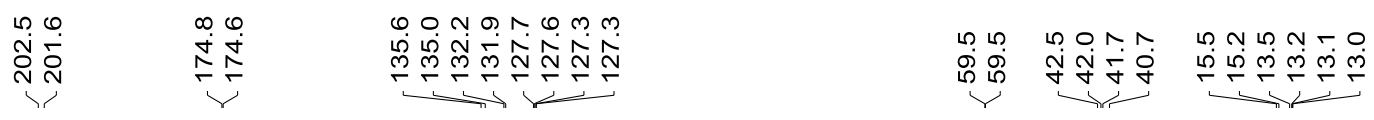

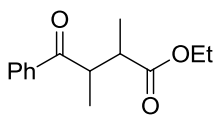

${ }^{13} \mathrm{C} \mathrm{NMR}\left(100 \mathrm{MHz}, \mathrm{CDCl}_{3}\right)$ of $\mathbf{3 k}$

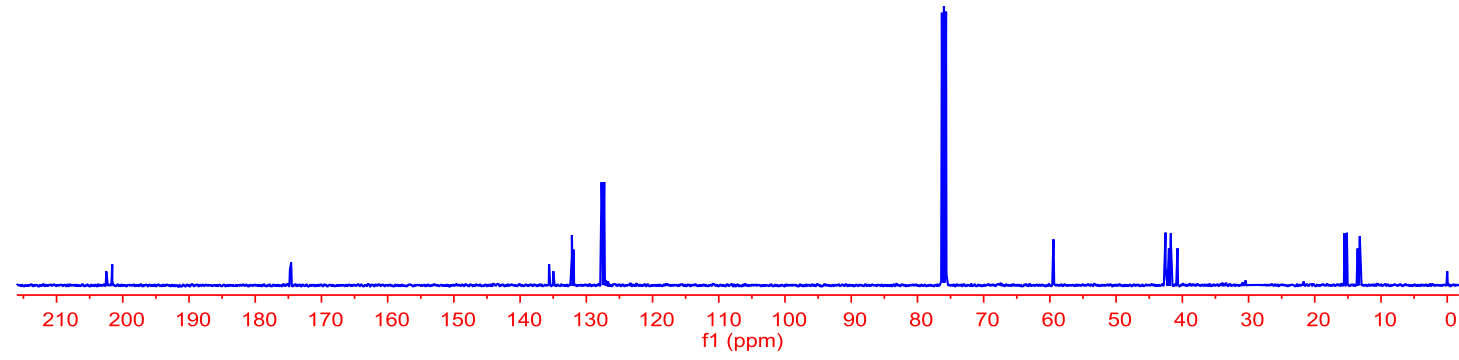

\section{œ}

NヘN

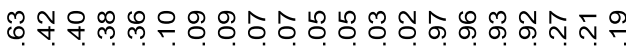

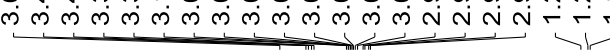

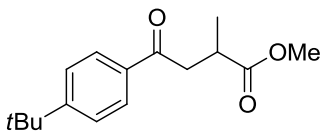

${ }^{1} \mathrm{H} \mathrm{NMR}\left(400 \mathrm{MHz}, \mathrm{CDCl}_{3}\right)$ of $4 \mathrm{a}$

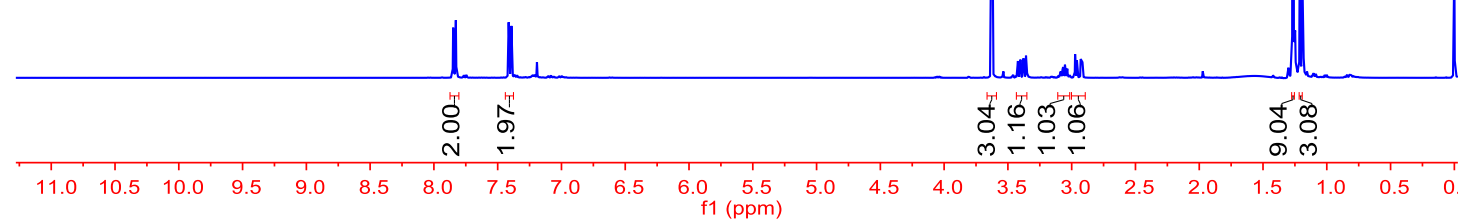




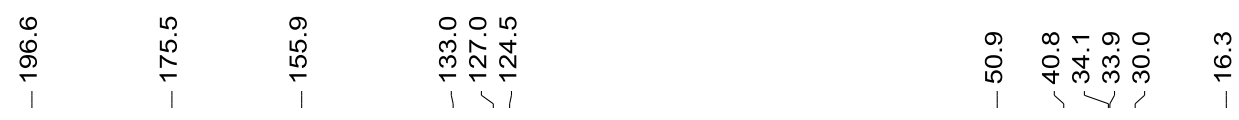

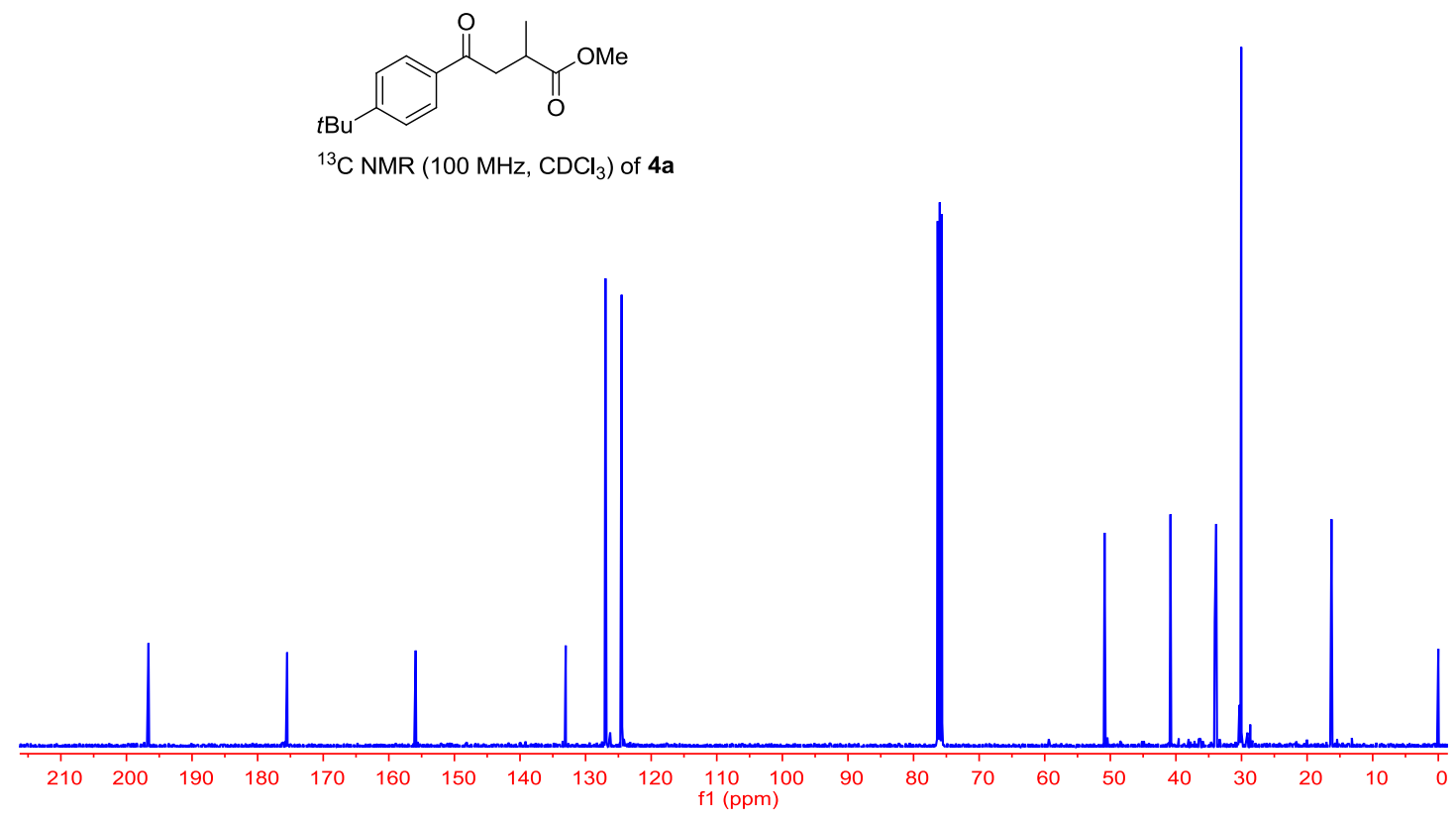

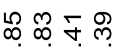

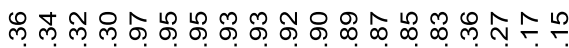

Nin

ต่

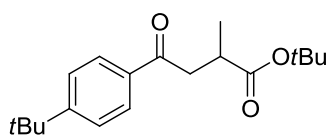

${ }^{1} \mathrm{H} \mathrm{NMR}\left(400 \mathrm{MHz}, \mathrm{CDCl}_{3}\right)$ of $\mathbf{4 b}$

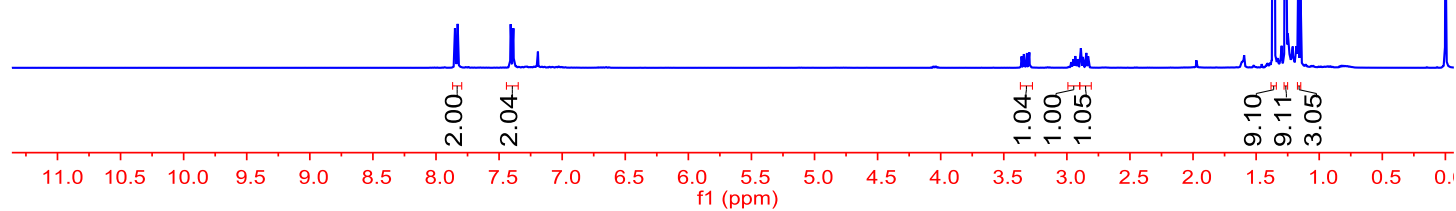




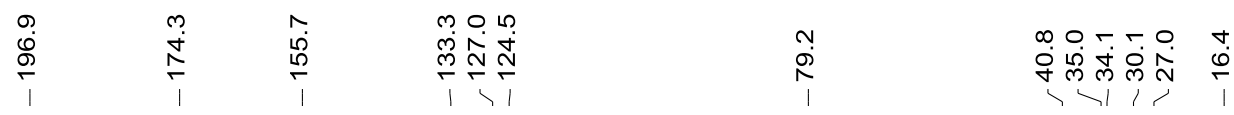

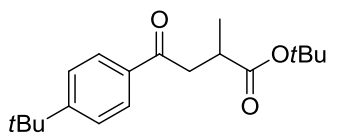

${ }^{13} \mathrm{C} \mathrm{NMR}\left(100 \mathrm{MHz}, \mathrm{CDCl}_{3}\right)$ of $\mathbf{4 b}$

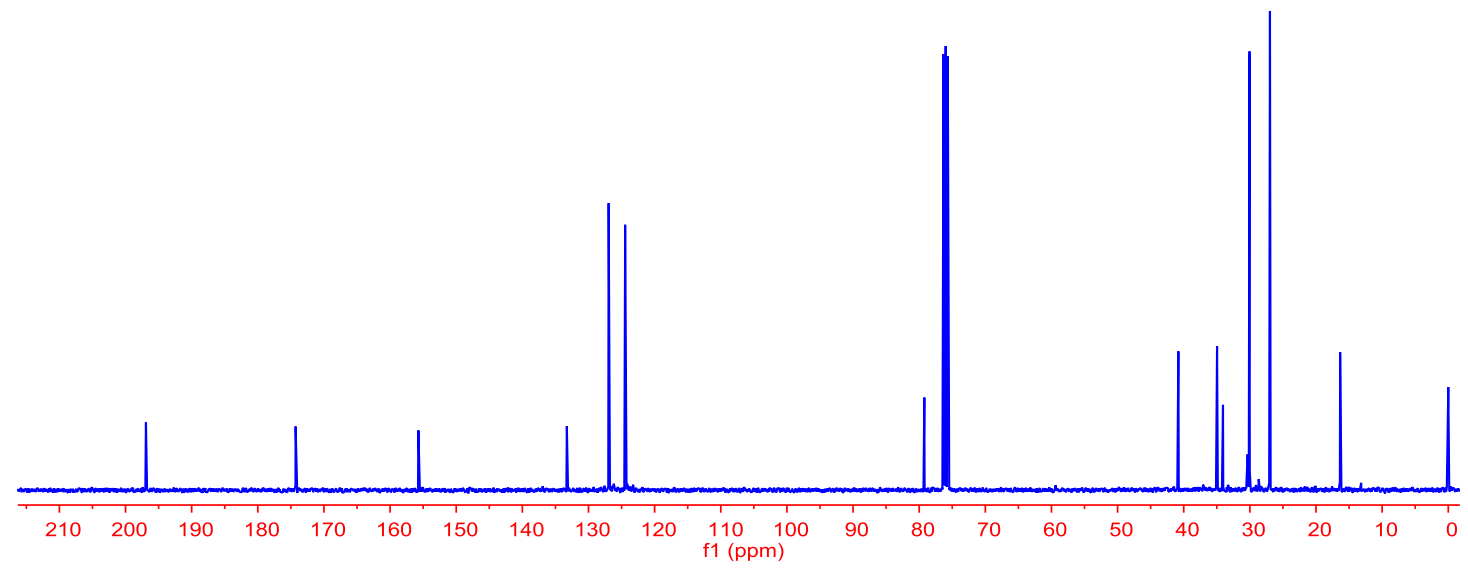

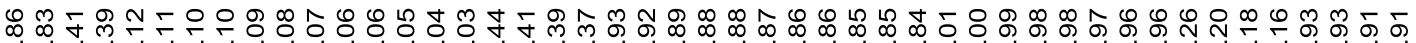

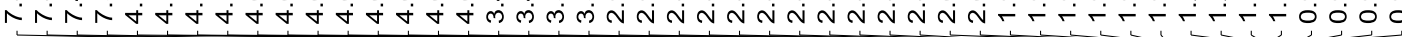

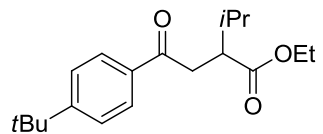

${ }^{1} \mathrm{H} \mathrm{NMR}\left(400 \mathrm{MHz}, \mathrm{CDCl}_{3}\right)$ of $4 \mathrm{c}$

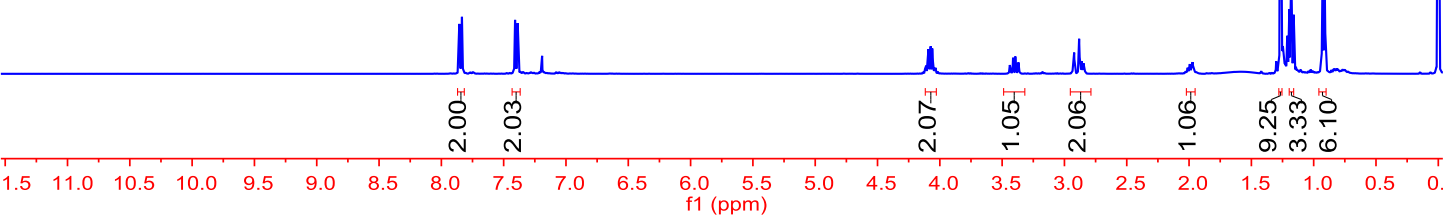




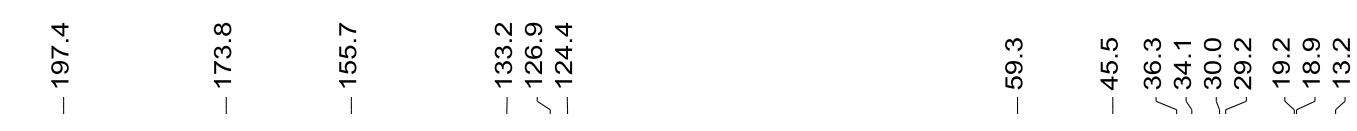<smiles>CCOC(=O)C(CC(=O)c1ccc(C(C)(C)C)cc1)C(C)C</smiles>

${ }^{13} \mathrm{C} \mathrm{NMR}\left(100 \mathrm{MHz}, \mathrm{CDCl}_{3}\right)$ of $4 \mathrm{c}$

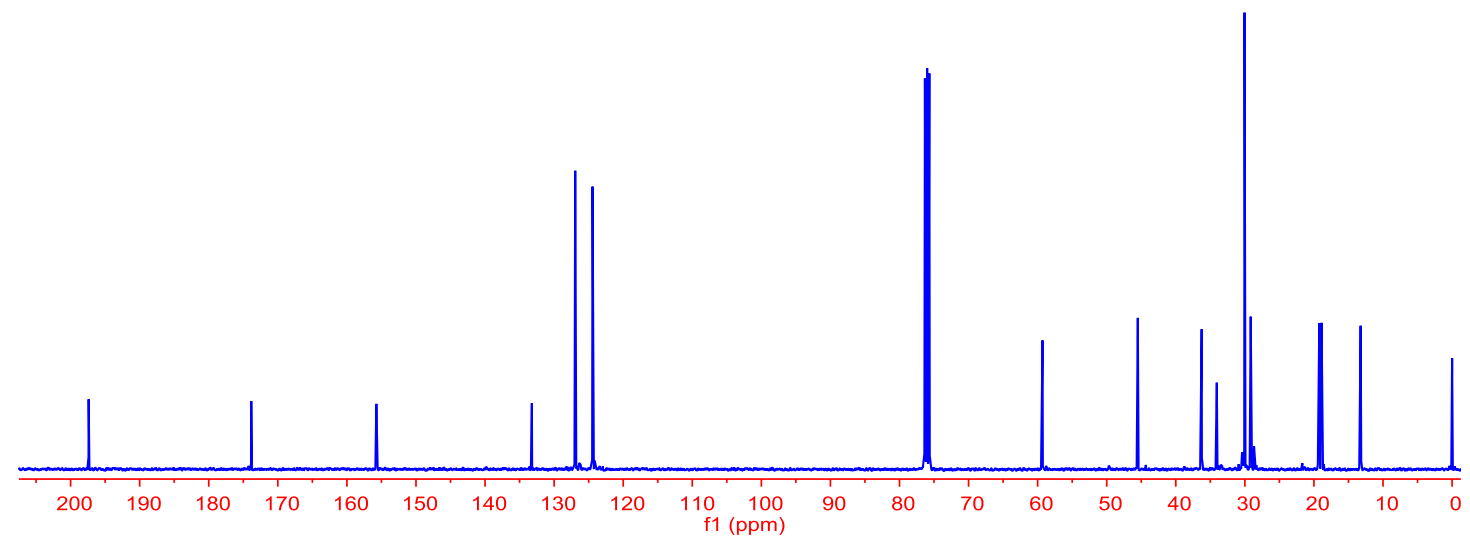

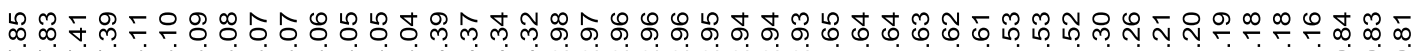
rín<smiles>CCOC(=O)C(CC(=O)c1ccc(C(C)(C)C)cc1)C(C)C</smiles>

${ }^{1} \mathrm{H} \mathrm{NMR}\left(400 \mathrm{MHz}, \mathrm{CDCl}_{3}\right)$ of $\mathbf{4 d}$

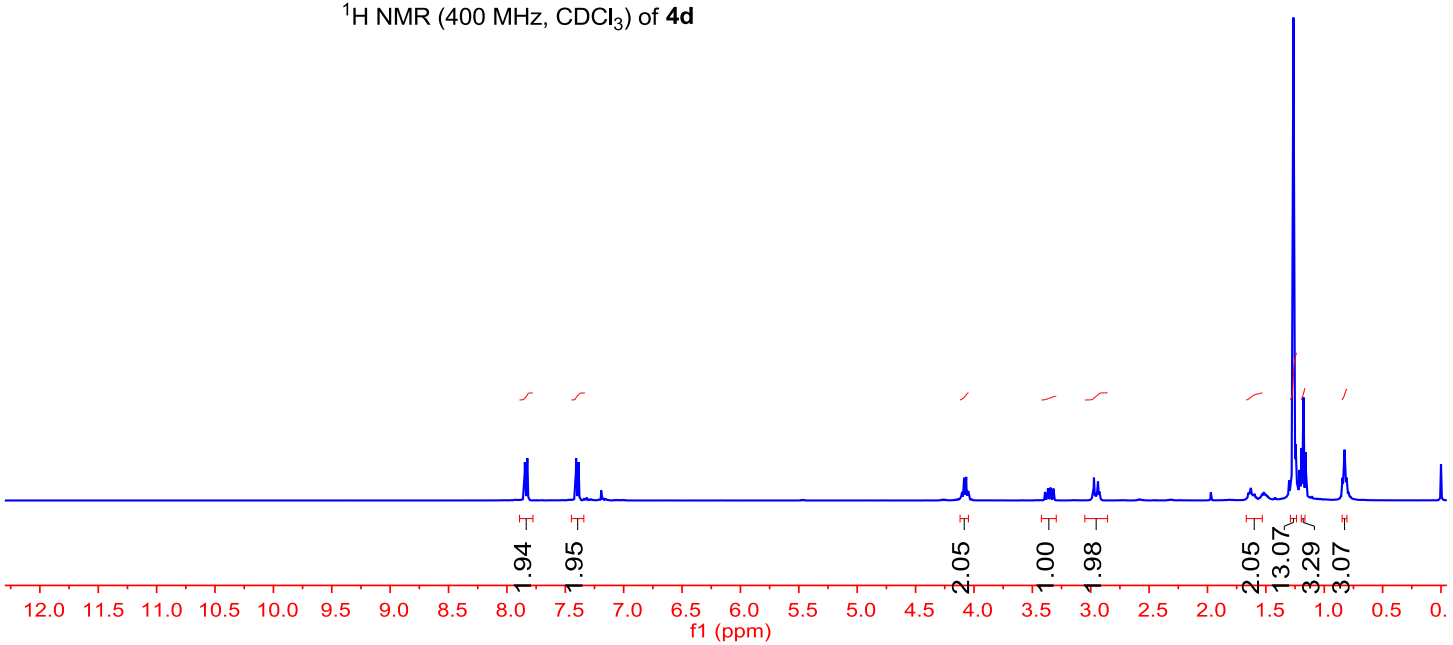




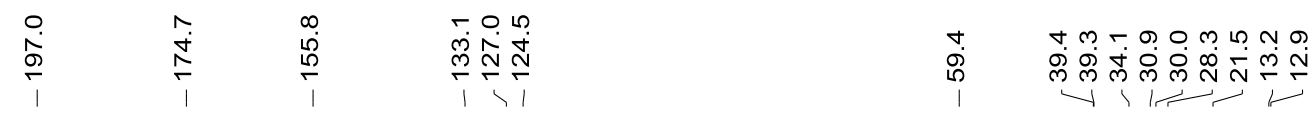<smiles>CCOC(=O)C(CC(=O)c1ccc(C(C)(C)C)cc1)C(C)C</smiles>

${ }^{13} \mathrm{C} \mathrm{NMR}\left(100 \mathrm{MHz}, \mathrm{CDCl}_{3}\right)$ of $4 \mathrm{~d}$

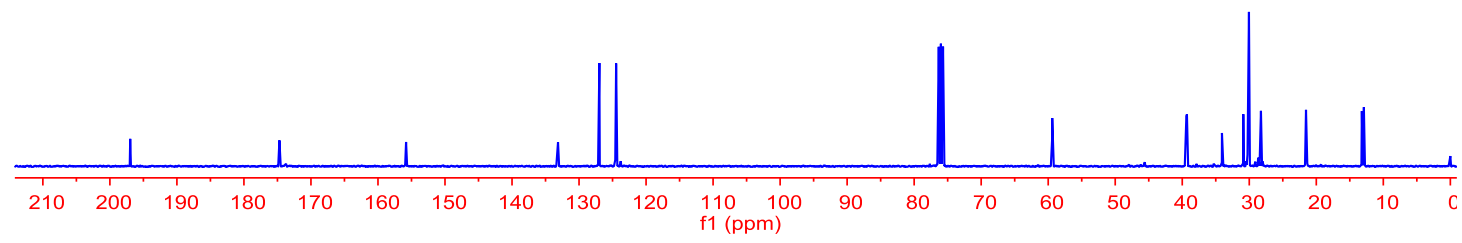

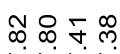

rín

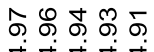

ن

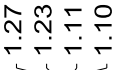

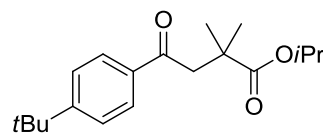

${ }^{1} \mathrm{H}$ NMR (400 MHz, $\mathrm{CDCl}_{3}$ ) of $4 \mathrm{e}$

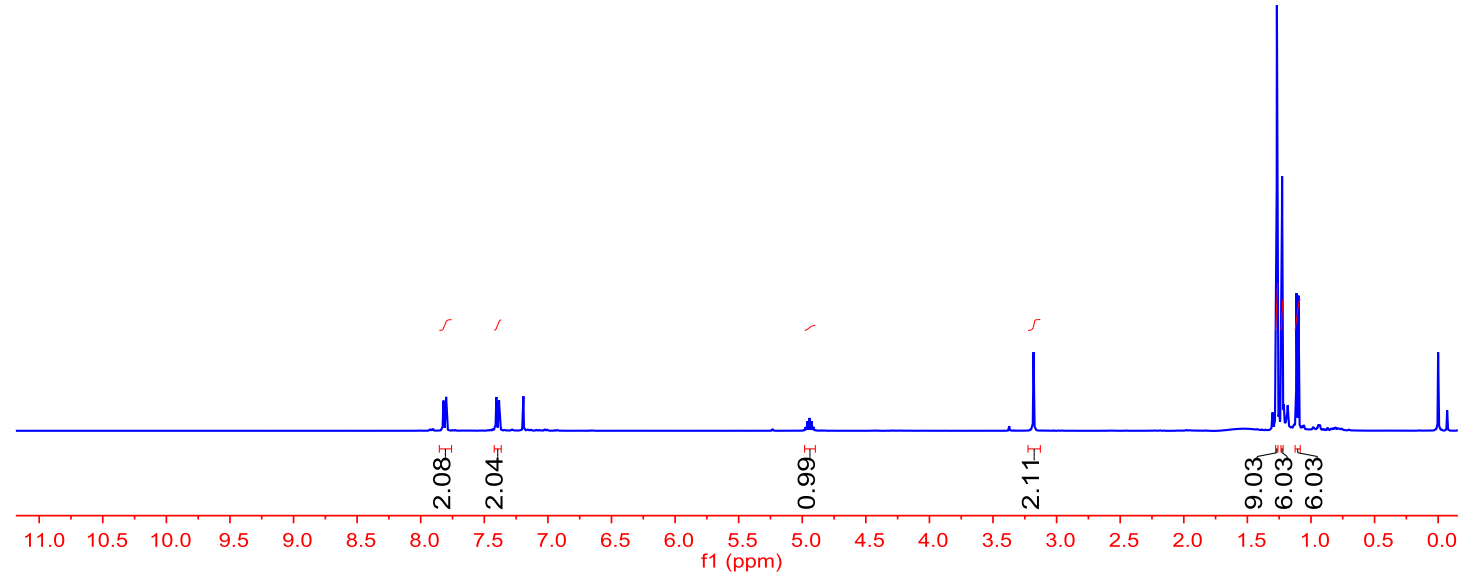




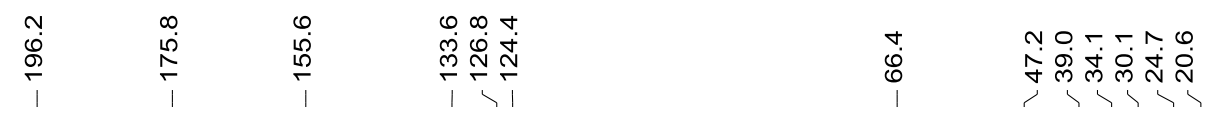

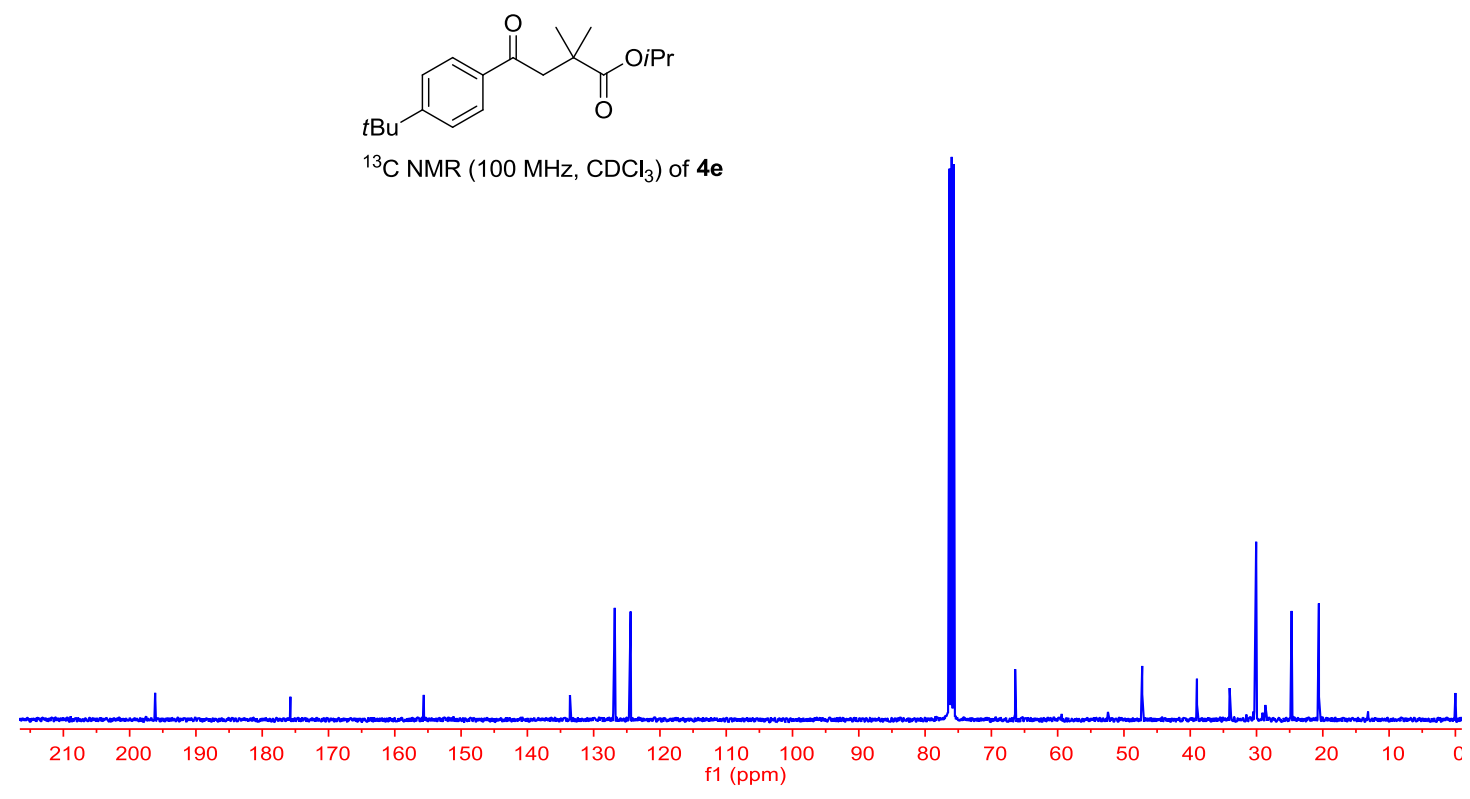

œ

NNN

คำ స్

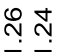

i

ì

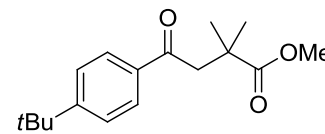

${ }^{1} \mathrm{H}$ NMR (400 MHz, $\mathrm{CDCl}_{3}$ ) of $\mathbf{4 f}$

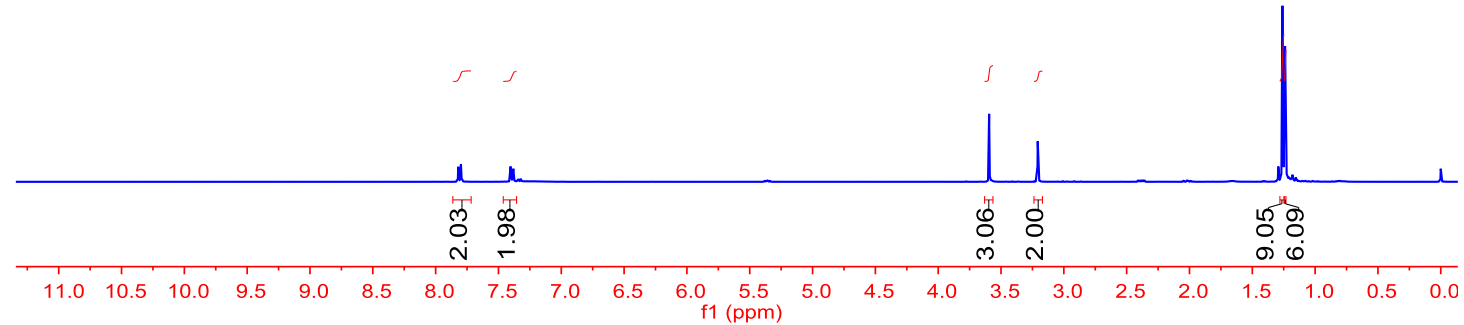




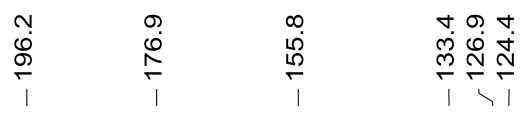

0 L $0.0 \%$

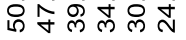

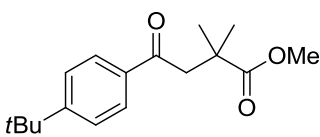

${ }^{13} \mathrm{C}$ NMR $\left(100 \mathrm{MHz}, \mathrm{CDCl}_{3}\right)$ of $\mathbf{4 f}$

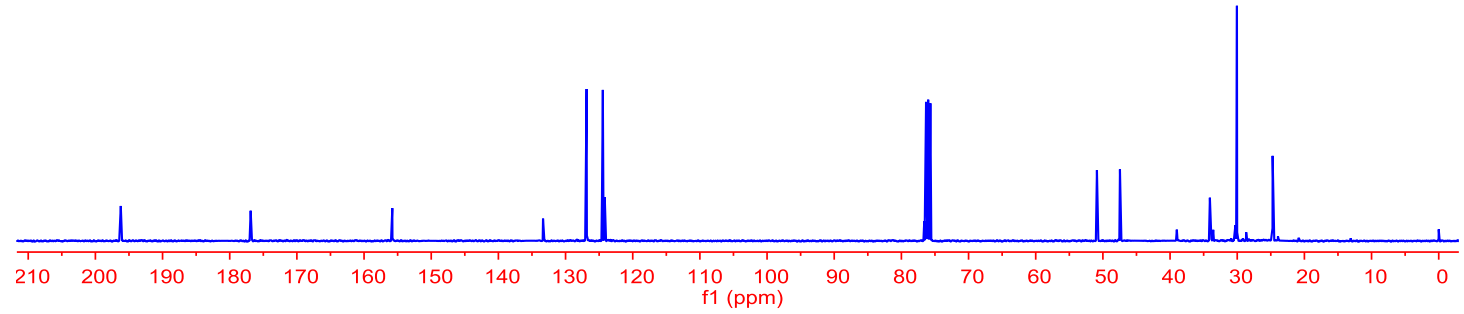

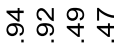

iñ

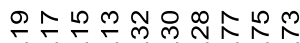

隹

r

(-

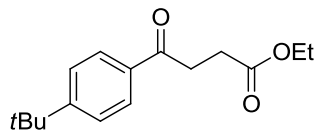

${ }^{1} \mathrm{H}$ NMR $\left(400 \mathrm{MHz}, \mathrm{CDCl}_{3}\right)$ of $\mathbf{4 g}$

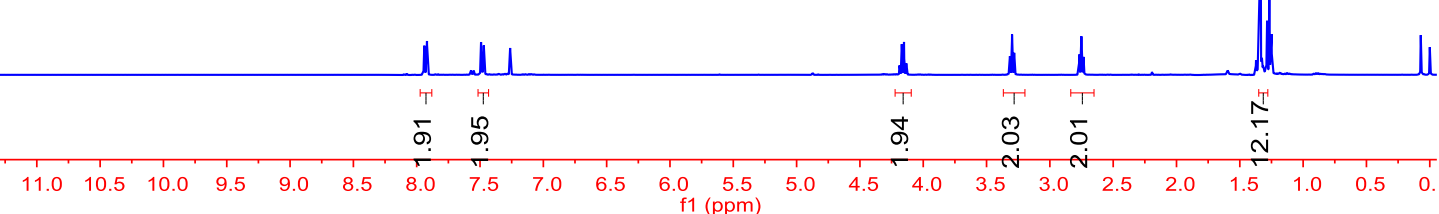




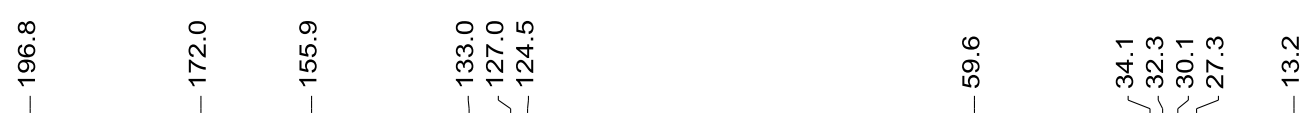<smiles>CCOC(=O)CCC(=O)c1ccc(C(C)(C)C)cc1</smiles>

${ }^{13} \mathrm{C} \mathrm{NMR} \mathrm{(100} \mathrm{MHz,} \mathrm{CDCl}_{3}$ ) of $\mathbf{4 g}$

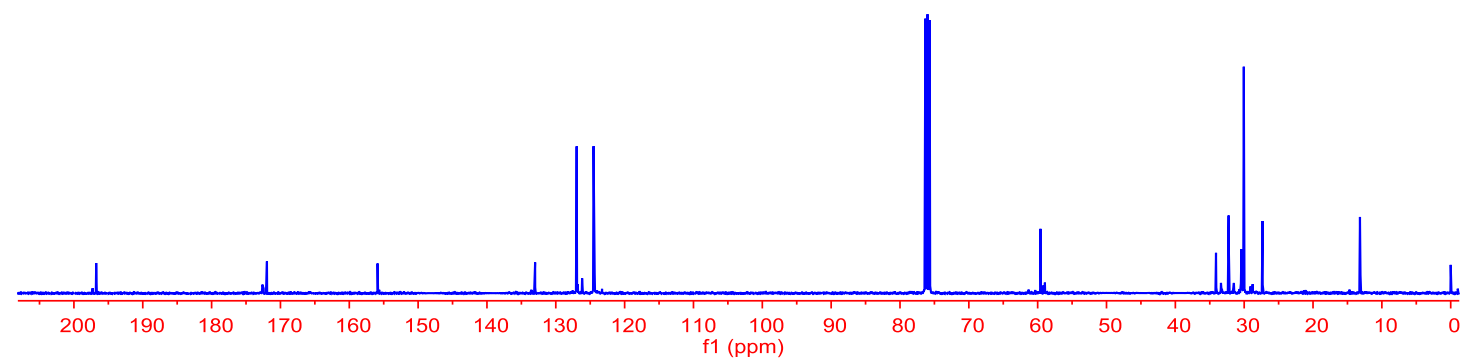

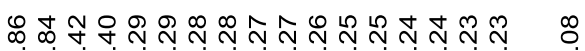

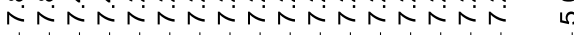

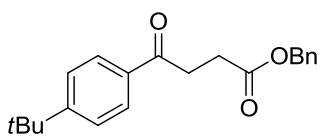

${ }^{1} \mathrm{H} \mathrm{NMR}\left(400 \mathrm{MHz}, \mathrm{CDCl}_{3}\right)$ of $\mathbf{4 h}$

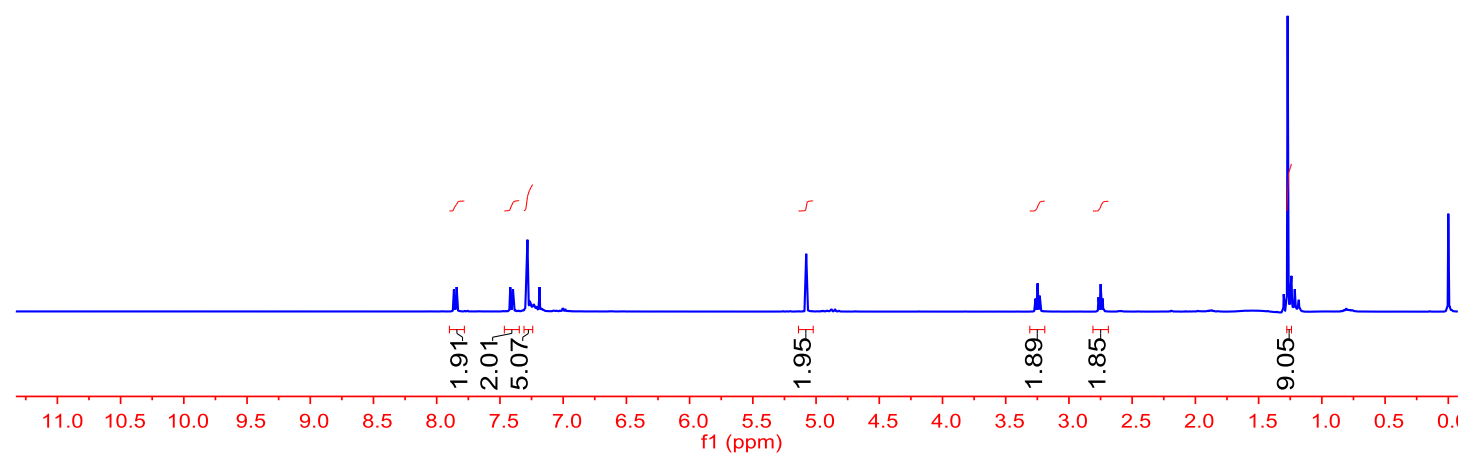




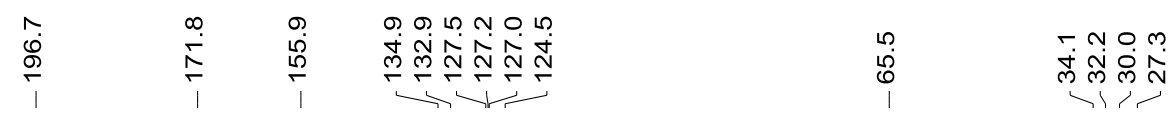

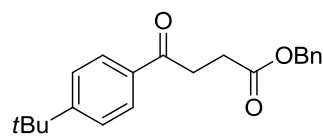

${ }^{13} \mathrm{C} \mathrm{NMR}\left(100 \mathrm{MHz}, \mathrm{CDCl}_{3}\right)$ of $\mathbf{4 h}$

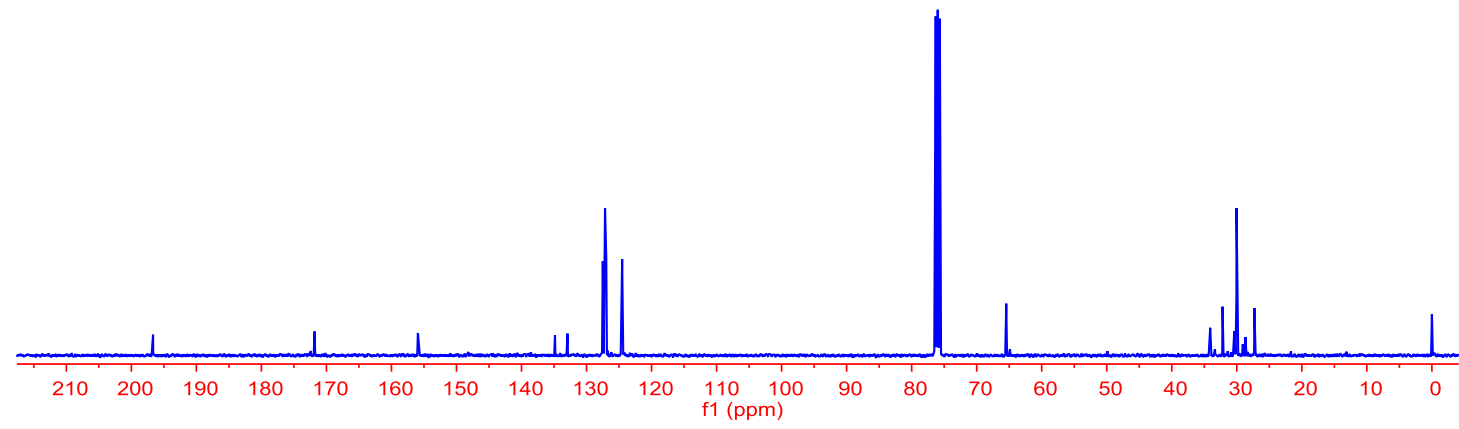

ભ

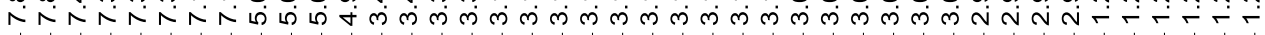<smiles>CC(=O)OCCCCC(C)CC(=O)c1ccc(C(C)(C)C)cc1</smiles>

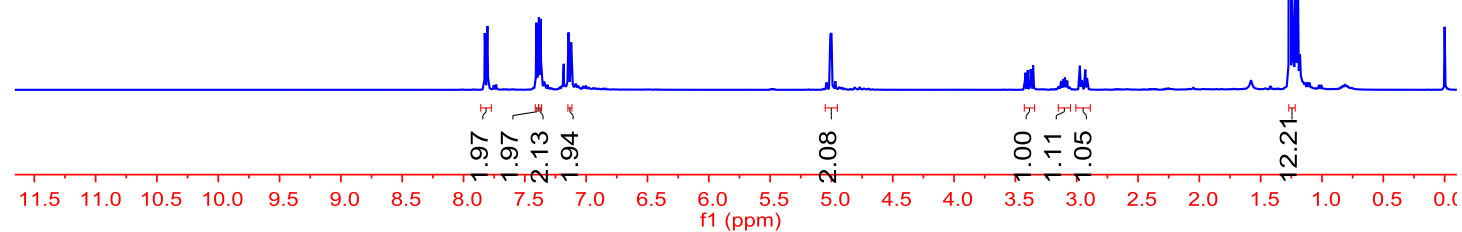




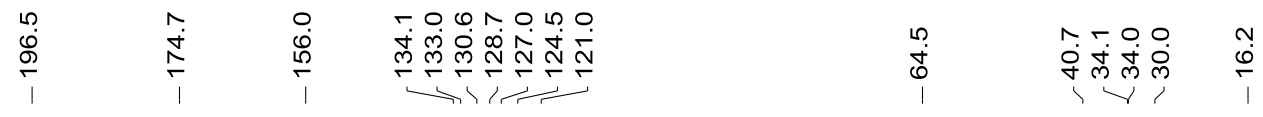<smiles>CC(=O)OCC(C)C(=O)OCc1ccc(Br)cc1</smiles>

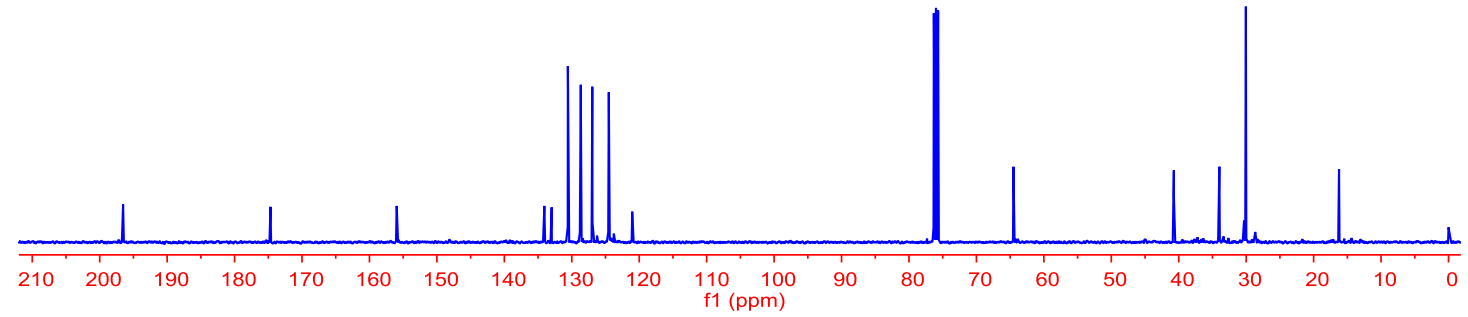

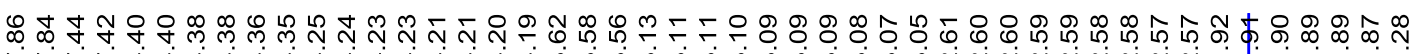

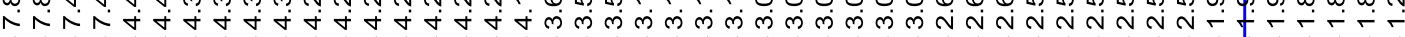<smiles>CC(C)(C)c1ccc(C(=O)CC2CCOC2=O)cc1</smiles>

${ }^{1} \mathrm{H} \mathrm{NMR}\left(400 \mathrm{MHz}, \mathrm{CDCl}_{3}\right)$ of $\mathbf{4 j}$

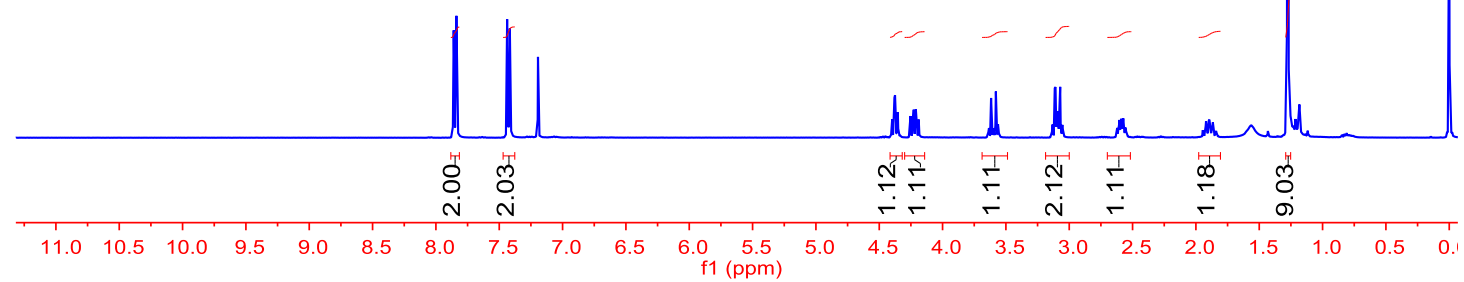




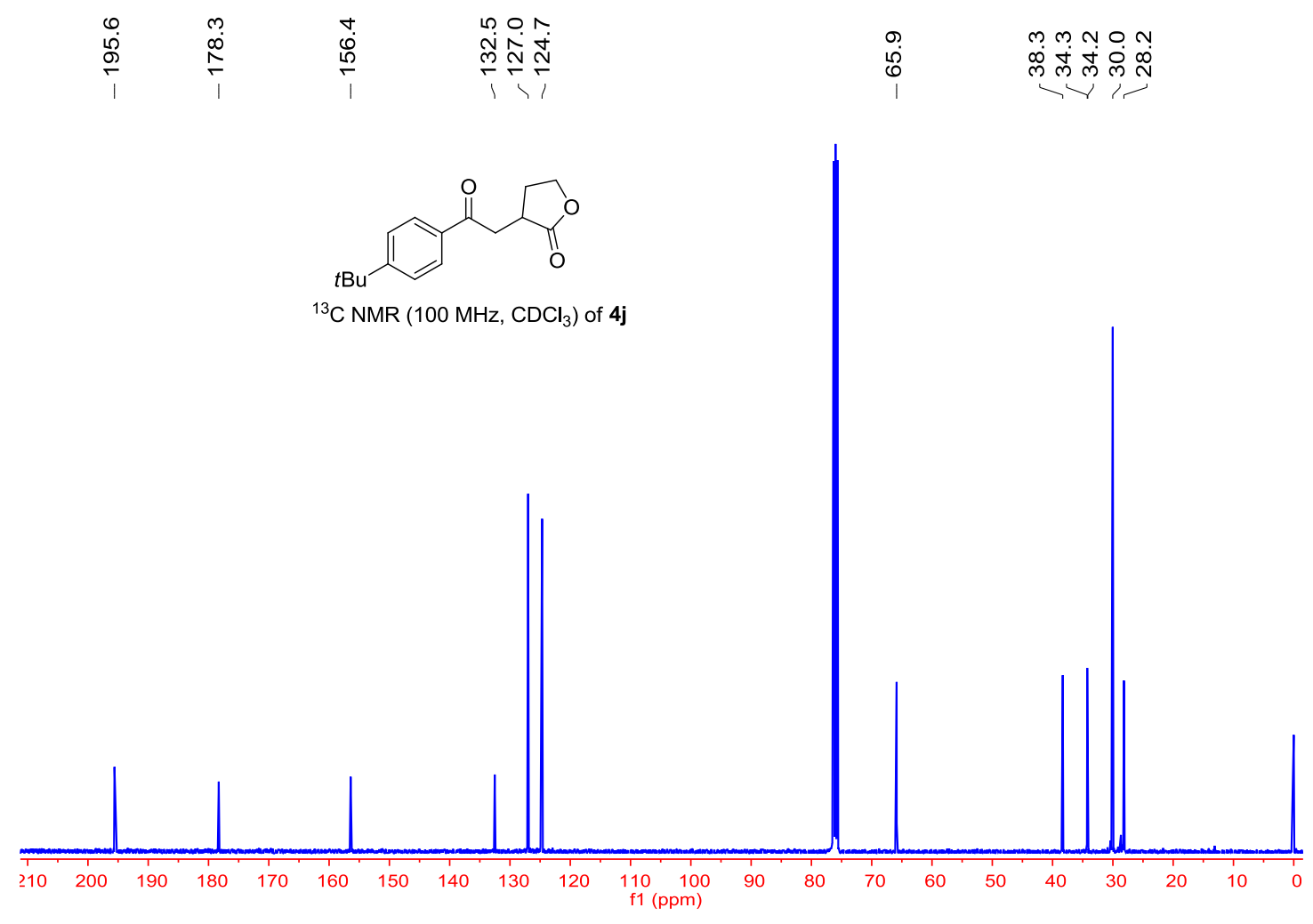

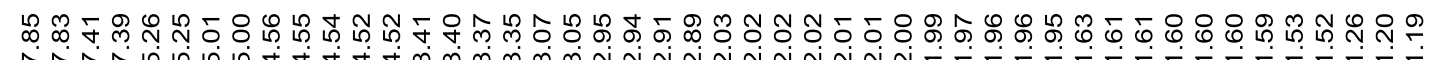
viñ
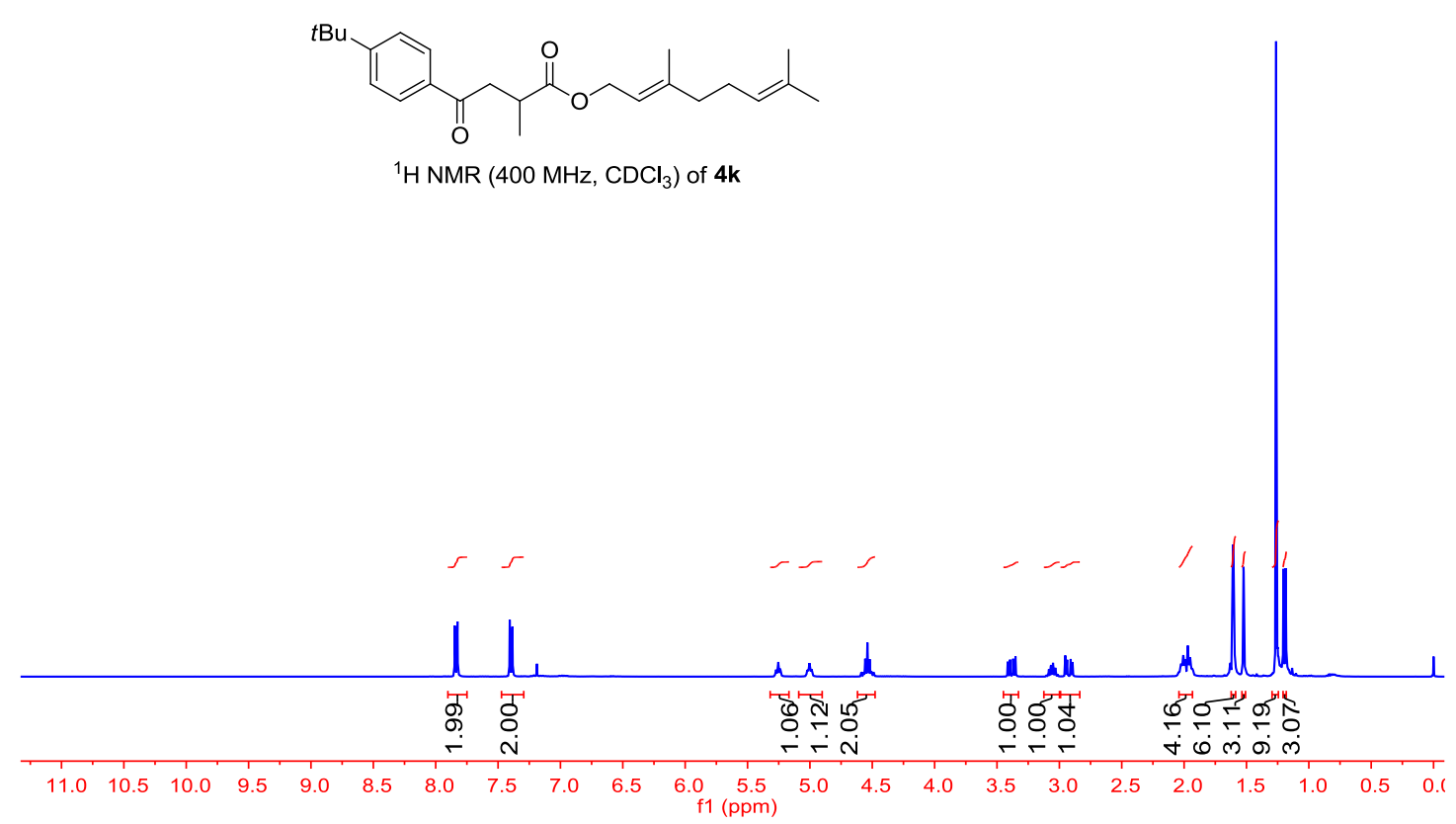

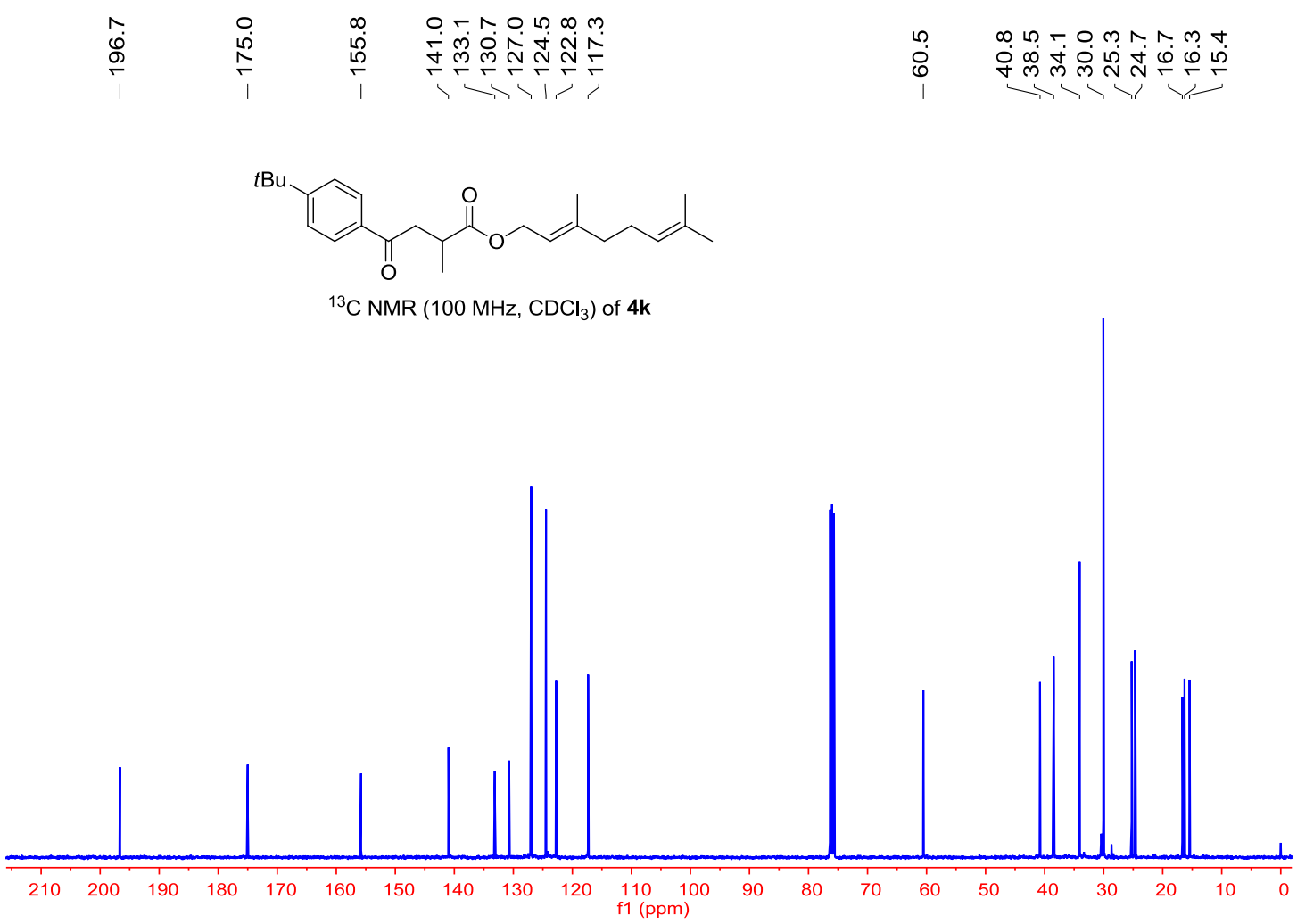

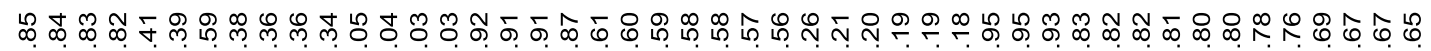
ヘ N N N<smiles>CC(C)C[C@H]1CCC(C(C)C)CC1OC(=O)C(C)CC(=O)c1ccc(C(C)(C)C)cc1</smiles>

${ }^{1} \mathrm{H} \mathrm{NMR}\left(400 \mathrm{MHz}, \mathrm{CDCl}_{3}\right)$ of $4 \mathrm{I}$

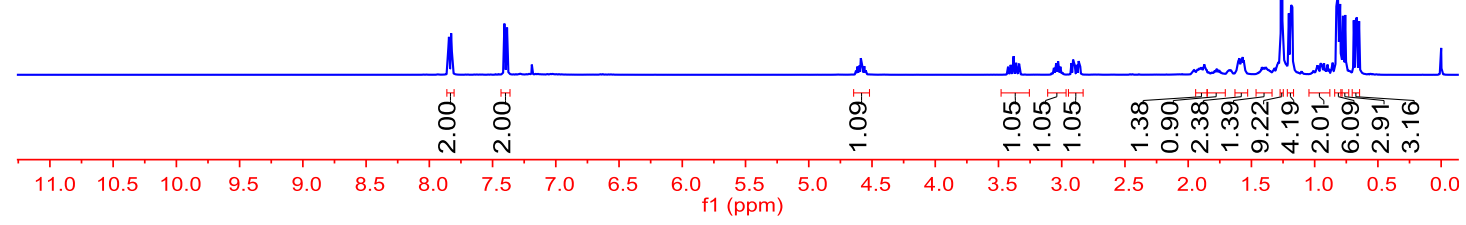




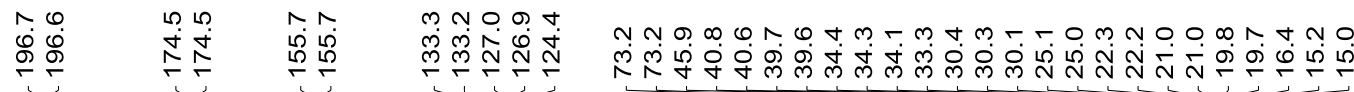

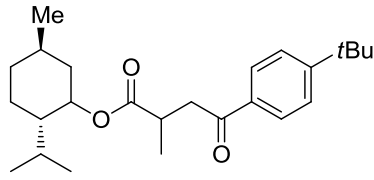

${ }^{13} \mathrm{C} \mathrm{NMR}\left(100 \mathrm{MHz}, \mathrm{CDCl}_{3}\right)$ of $4 \mathrm{I}$

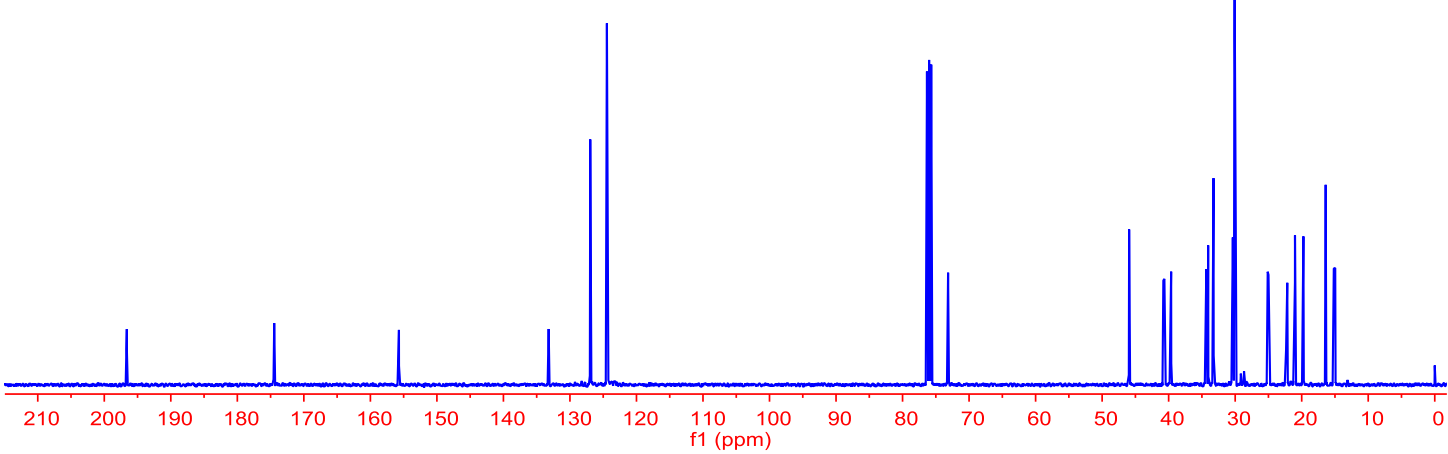

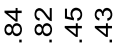

NiN

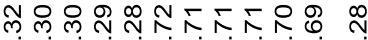

mmm

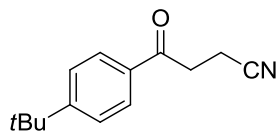

$\left.{ }^{1} \mathrm{H} \mathrm{NMR} \mathrm{(400} \mathrm{MHz,} \mathrm{CDCl}_{3}\right)$ of $\mathbf{5 a}$

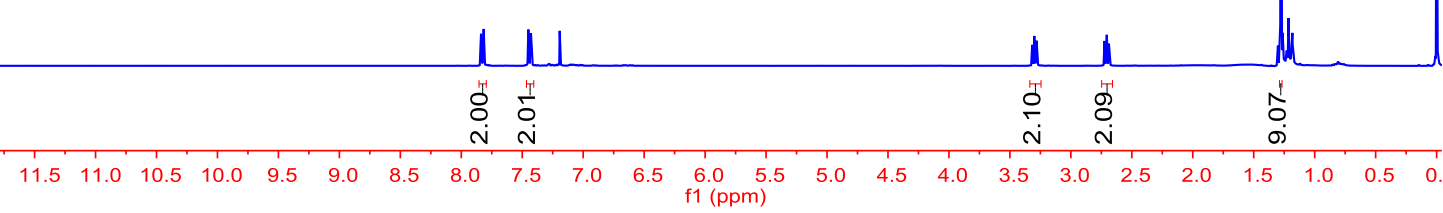


<smiles>CC(C)(C)c1ccc(C(=O)CCC#N)cc1</smiles>

${ }^{13} \mathrm{C} \mathrm{NMR}\left(100 \mathrm{MHz}, \mathrm{CDCl}_{3}\right)$ of $\mathbf{5 a}$

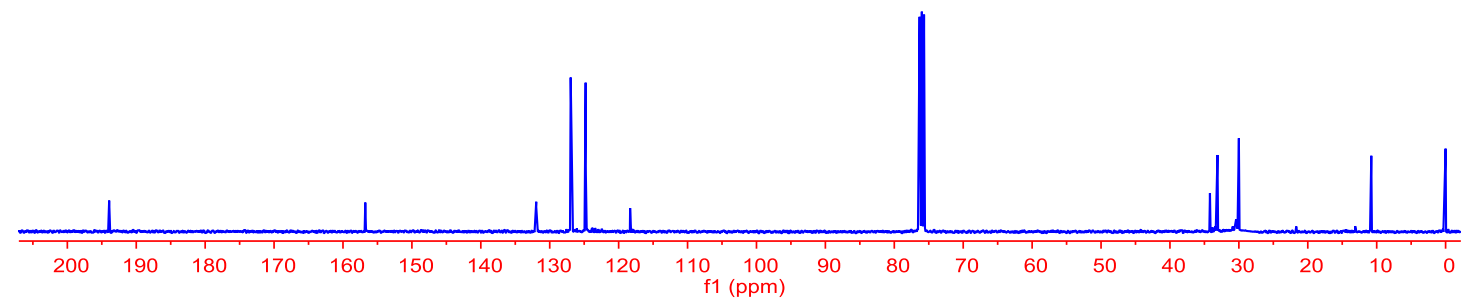

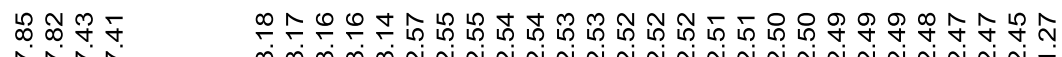

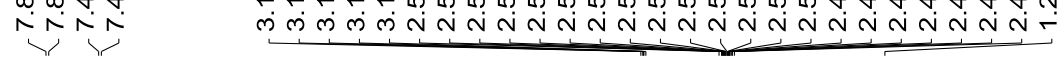<smiles>CC(C)(C)c1ccc(C(=O)CCC(F)(F)F)cc1</smiles>

${ }^{1} \mathrm{H} \mathrm{NMR}\left(400 \mathrm{MHz}, \mathrm{CDCl}_{3}\right)$ of $\mathbf{5 b}$

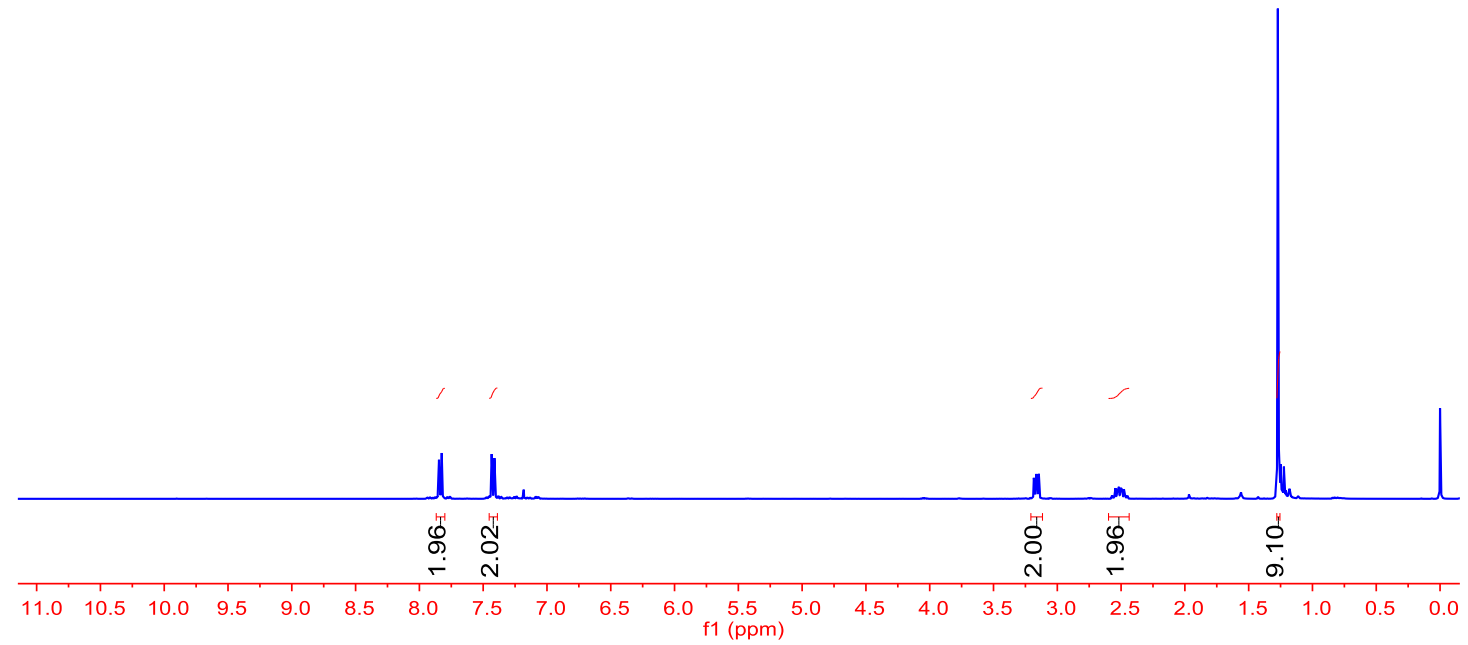



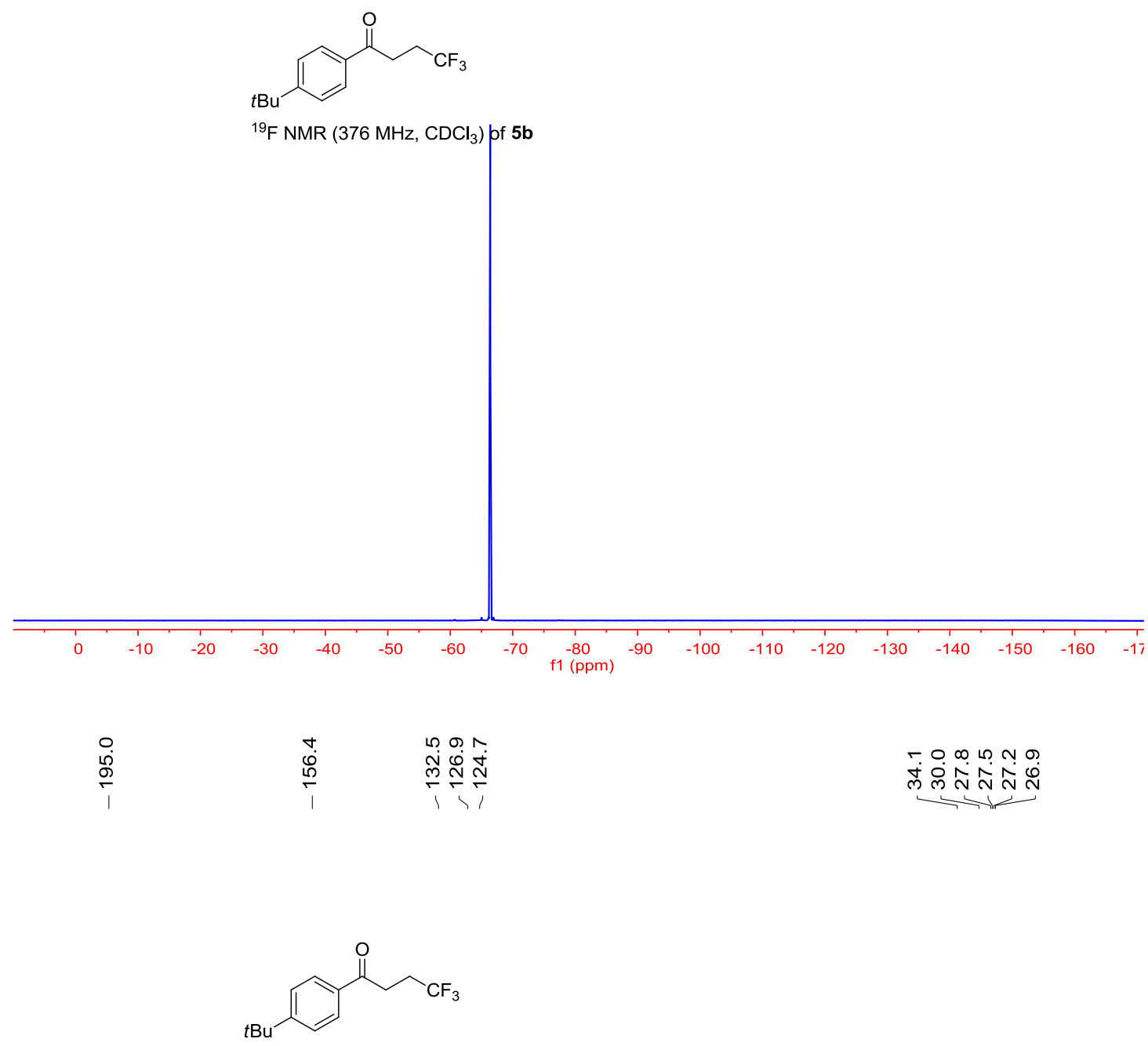

${ }^{13} \mathrm{C} \mathrm{NMR}\left(100 \mathrm{MHz}, \mathrm{CDCl}_{3}\right)$ of $\mathbf{5 b}$

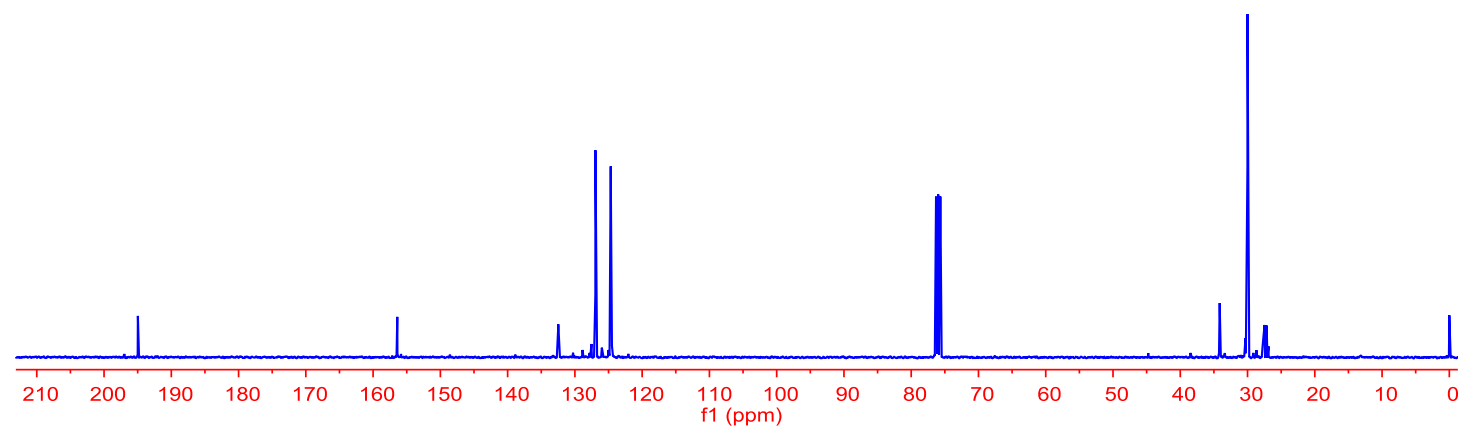




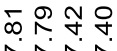

NNN

:

$\stackrel{\infty}{\stackrel{\infty}{r}} \stackrel{\stackrel{\infty}{r}}{\stackrel{\Gamma}{+}}$

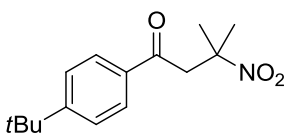

${ }^{1} \mathrm{H}$ NMR $\left(400 \mathrm{MHz}, \mathrm{CDCl}_{3}\right)$ of $5 \mathrm{c}$

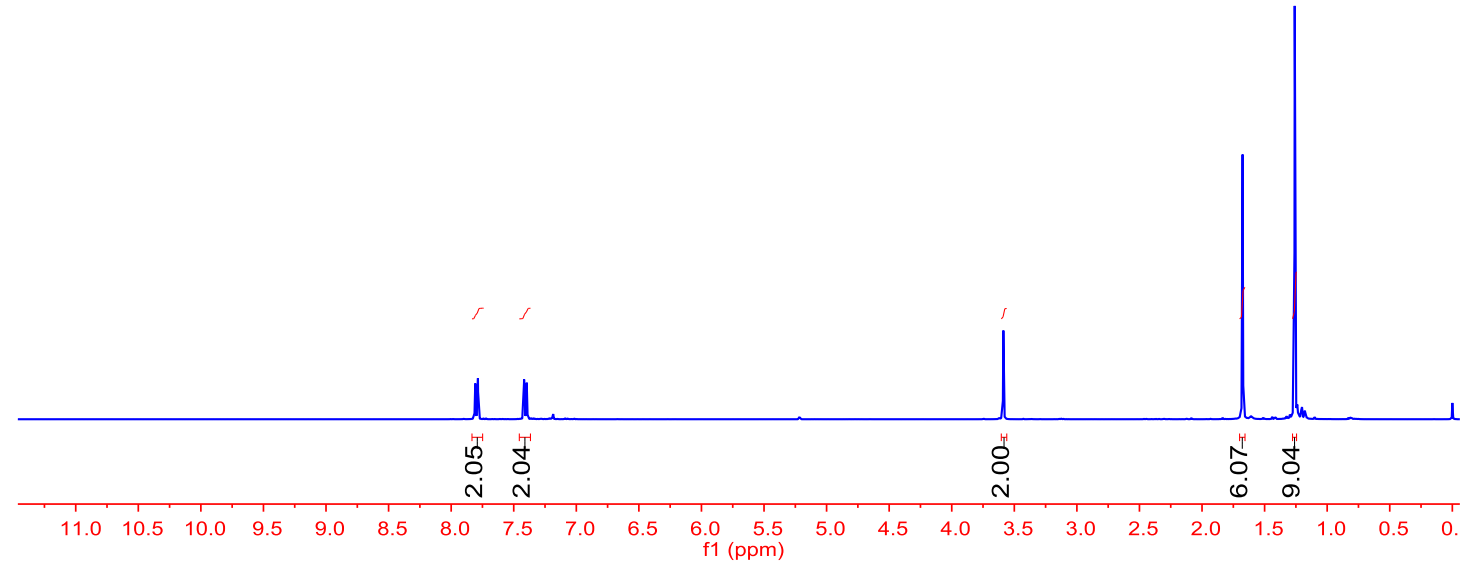

\begin{tabular}{|c|c|c|}
\hline$\frac{5}{i n}$ & 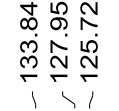 & 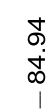 \\
\hline
\end{tabular}

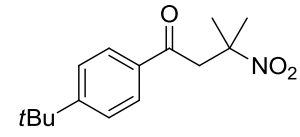

${ }^{13} \mathrm{C}$ NMR $\left(100 \mathrm{MHz}, \mathrm{CDCl}_{3}\right)$ of $\mathbf{5 c}$

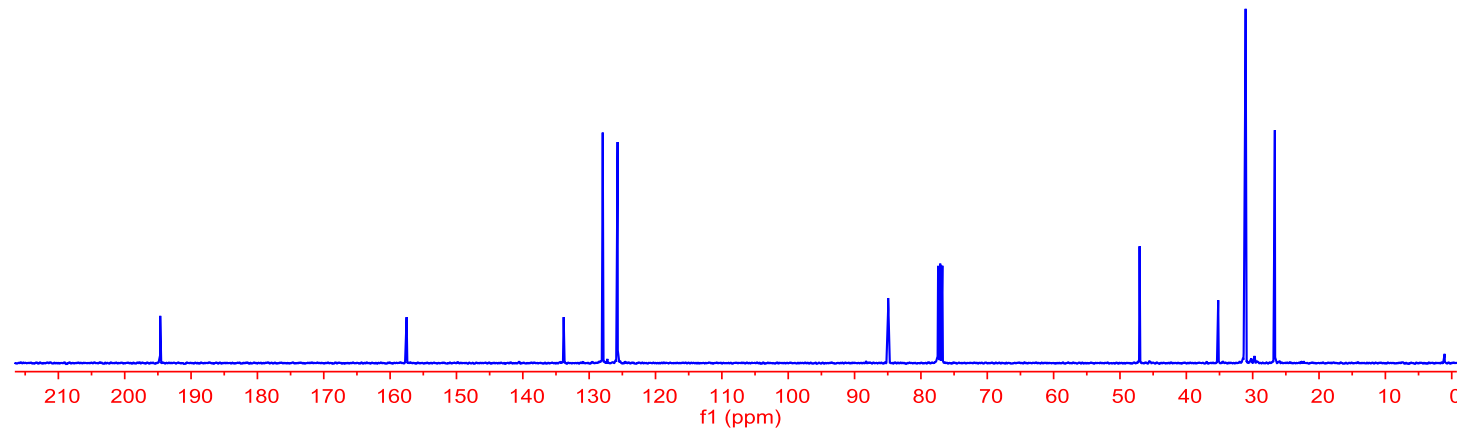




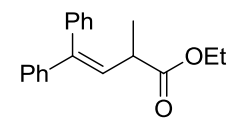

${ }^{1} \mathrm{H} \mathrm{NMR} \mathrm{(400} \mathrm{MHz,} \mathrm{CDCl}_{3}$ ) of 6

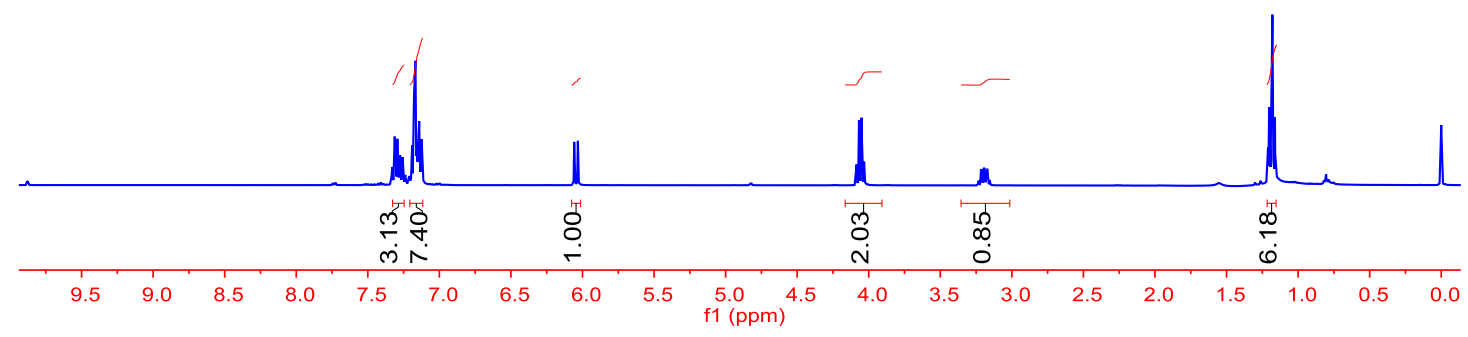

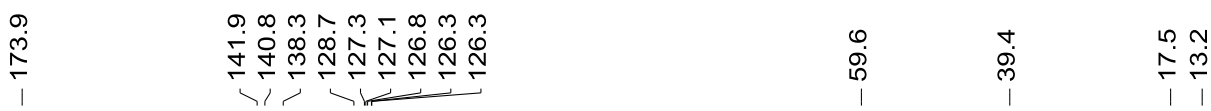

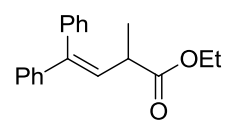

${ }^{13} \mathrm{C} \mathrm{NMR}\left(100 \mathrm{MHz}, \mathrm{CDCl}_{3}\right)$ of 6

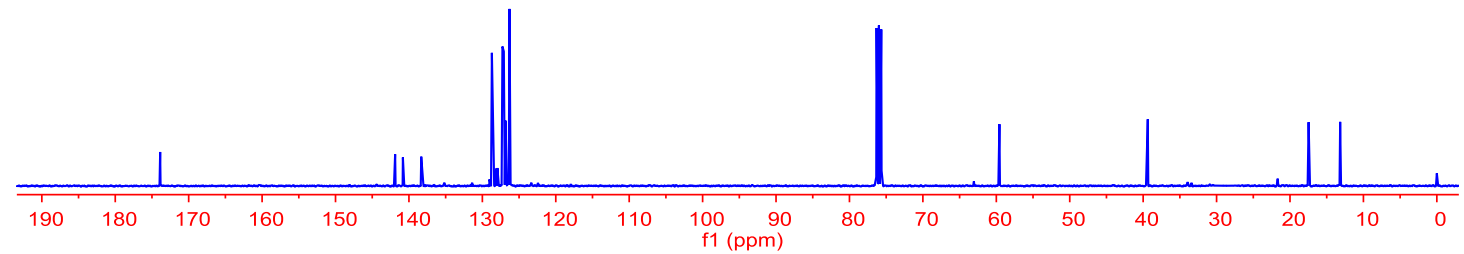

